\title{
La surveillance bancaire belge de 1935 façonnée sur le modèle suisse: un discret transfert de politique publique ? Thibaud Giddey
}

\begin{abstract}
The 1935 Belgian Banking Control System Inspired by the Swiss Model : a Discrete Policy Transfer?

Following the severe financial crisis that shook most of Western societies in the early 1930s, a strong shift towards state regulation on banking activities took place in numerous countries. Among them, both Belgium and Switzerland undertook the implementation of banking laws. More precisely, the Swiss Federal Banking Law of November 1934 was enacted a few months before the Belgian Royal Decree Nr. 185 of July 1935. While the historical context and the development of each of these regulations have been studied individually, a comparative perspective is still lacking. Moreover, the question of the possible links between these two regulatory regimes remains largely unexplored. A detailed analysis of the Belgian banking regulation process shows that the Swiss law acted as a role model, put forward by the representatives of the banking circles. The Swiss influence played a significant part in shaping the banking regulation in a liberal perspective ; thus meeting two essential needs for the Belgian bankers : to limit state intervention to a minimum, and to bypass the authority of the central bank. This relationship between banking laws finds its counterpart in the activities of the two supervision agencies set up in the 1930s, the 'Commission fédérale des banques' in Berne and the 'Commission bancaire' in Brussels. Both bodies featured limited means of actions and experienced similar paths during their first years of operation.
\end{abstract}

\section{Citer ce document / Cite this document :}

Giddey Thibaud. La surveillance bancaire belge de 1935 façonnée sur le modèle suisse: un discret transfert de politique publique ?. In: Revue belge de philologie et d'histoire, tome 92, fasc. 4, 2014. Histoire médiévale, moderne et contemporaine Middeleeuwse, moderne en hedendaagse geschiedenis. pp. 1211-1243;

doi : https://doi.org/10.3406/rbph.2014.8608

https://www.persee.fr/doc/rbph_0035-0818_2014_num_92_4_8608

Fichier pdf généré le 18/04/2018 


\title{
La surveillance bancaire belge de 1935 façonnée sur le modèle suisse: un discret transfert de politique publique?
}

\author{
Thibaud GIDDEY \\ Université de Lausanne
}

\begin{abstract}
«L'état de nécessité qui a suscité en Suisse l'institution d'un régime de contrôles, parmi lesquels celui des banques, est fort voisin de la situation étranglée qui, dans notre pays, a amené le gouvernement, à intervenir dans un sens parallèle. Puisqu'il faut aux mêmes maladies appliquer les mêmes remèdes et que nécessité fait loi, il convient donc de s'inspirer du traitement qui paraît le meilleur.»
\end{abstract}

Le Pour et le Contre, journal financier belge, 14 avril 1935

Quels principes président à l'élaboration d'un régime de régulation? Chez les législateurs, quels sont les facteurs déterminants dans les choix institutionnels? Peut-on parler de transfert transnational de politiques publiques dans le contexte de la crise des années 1930? Cette contribution apporte quelques éléments de réponse à ces interrogations en abordant la thématique de la surveillance bancaire.

En juillet 1935, le gouvernement belge promulgua un arrêté royal sur le contrôle bancaire. Cette réglementation définit les bases d'un système de régulation bancaire maintenu, malgré diverses modifications secondaires, pendant plus d'un demi-siècle. Quelques mois plus tôt, en novembre 1934, le parlement suisse adoptait une loi sur les banques et les caisses d'épargne, première réglementation bancaire du pays à l'échelle fédérale. Les deux lois bancaires, leur élaboration et leurs qualités respectives ont déjà fait l'objet d'un certain nombre d'études, sur lesquels nous reviendrons. En revanche, très peu d'historiens se sont penchés sur la comparaison entre les deux systèmes de surveillance et les influences que le modèle helvétique a eu chez les législateurs belges. L'objet de cet article est le suivant: après avoir établi les liens de parenté entre les lois suisse et belge et mesuré l'influence helvétique, il s'agira de comprendre les raisons qui ont fait de la loi suisse un modèle pour les législateurs belges. Nous tenterons également d'esquisser les similarités et différences notables qui résultent de cette parenté originale, en nous focalisant sur les deux autorités de surveillance mises en place dans chacun des pays - la Commission bancaire à Bruxelles et la Commission fédérale des banques à Berne ${ }^{(1)}$.

(1) Cet article se base sur des recherches effectuées dans le cadre d'une thèse en cours à l'Université de Lausanne sous la direction de Malik Mazbouri. Je remercie le Fonds national suisse de la Recherche Scientifique dont le soutien financier m'a permis d'effectuer des recherches dans les fonds d'archives belges.

Abréviations: $\mathrm{ABB}$ : Association belge des Banques; ABNB: Archives de la Banque na- 
Suite à la crise initiée en 2007, les questions de l'encadrement des activités financières par l'État ont connu un important regain d'intérêt, tant dans la recherche académique que dans le traitement médiatique. Les conséquences dramatiques de la crise ont en effet engendré la remise en question - parfois par les mêmes acteurs qui les avaient jusqu'alors promus - d'un certain nombre de crédos reposant sur la confiance absolue dans les mécanismes de discipline de marché et d'autorégulation ${ }^{(2)}$. Dans la recherche historique également, ces évolutions ont stimulé un réexamen des conditions d'émergence et de développement du contrôle bancaire. Tout en proposant de tirer les «leçons du passé» en s'adressant ainsi aux responsables politiques et économiques actuelles, ces études sont d'autre part souvent prisonnières des barrières nationales. Les spécificités propres au contexte national examiné permettant de justifier l'évolution singulière des établissements financiers et de leur surveillance. Parallèlement, dans le domaine de la science politique, les phénomènes de transfert de politique publique, d'émulation transnationale ou encore de lesson drawing se sont développés au cours des vingt dernières années, notamment sous l'influence de l'intégration européenne. Les chercheurs ont alors cherché à catégoriser et définir certaines typologies permettant de mieux analyser ces phénomènes de diffusion transnationale ${ }^{(3)}$.

Dans ce contexte théorique double, cet article propose de relire la naissance de la législation bancaire belge de 1935 à la lumière des influences internationales qui ont pu l'inspirer. Cette étude de cas belgo-helvétique permet ainsi non seulement de faire ressortir des caractéristiques nationales spécifiques, mais surtout de mettre en évidence quelques parallélismes frappants.

\section{La crise des années 1930 - catalyseur de la régulation bancaire}

La grande crise économique des années 1930, considérée par l'historien E.J. Hobsbawm comme le «plus grand tremblement de terre mondial jamais mesuré sur l'échelle de Richter des historiens de l'économie» ${ }^{(4)}$, constitue également le creuset dans lequel se sont forgées de nombreuses réformes politiques, économiques et sociales qui ont marqué la phase de croissance

tionale de Belgique, Bruxelles; AF: Archives fédérales, Berne; AGR: Archives générales du Royaume, Bruxelles; AGR2: Archives générales du Royaume 2 - dépôt Joseph Cuvelier, Bruxelles; ASG : Archives de la Société générale de Belgique, BNP-Paribas-Fortis Historical Centre, Bruxelles; BNB: Banque nationale de Belgique; BSGB: Banque de la Société générale de Belgique; IEV: Institut Émile Vandervelde, Bruxelles; OREC: Office de Redressement économique.

(2) Cf. par exemple: Nolan M. McCARTy, Keith T. Poole \& Howard Rosenthal, Political Bubbles. Financial Crises and the Failure of American Democracy, PrincetonOxford, Princeton University Press, 2013, p. 42-43; Joseph E. STIGLITZ, «The Financial Crisis of 2007-8 and its Macroeconomic Consequences », dans Stephany GRIFFITH-JONES, José Antonio OCAMPO \& Joseph E. STigliTz, eds., Time for a Visible Hand: Lessons from the 2008 World Financial Crisis, Oxford, Oxford University Press, 2010, p. 19-49.

(3) Sur le concept de policy transfer et ses évolutions récentes: David BENSON \& Andrew JORDAN, «What Have We Learned from Policy Transfer Research? Dolowitz and Marsh Revisited», dans Political Studies Review, t. 9, 2011, p. 366-378.

(4) Eric Hobsbawm, Age of Extremes. The Short Twentieth Century, 1914-1991, Londres, Michael Joseph, 1994, p. 86. 
des «Trente glorieuses». La composante bancaire et financière de la crise est, dans de nombreux pays, d'une telle ampleur, à la fois dans son rôle de détonateur et de diffusion des mécanismes de récession, qu'elle donne souvent lieu, de la part des autorités publiques, à des renforcements des réglementations ${ }^{(5)}$. Ainsi, à l'échelle mondiale, la période des années 1930 est marquée par un véritable foisonnement de législations ayant pour objet de réglementer les opérations bancaires. Face à l'absence de synthèses historiques récentes retraçant ce mouvement international de régulation bancaire, les publications d'époque, souvent des études de droit comparé ou de politique économique comparée, sont des sources utiles pour apprécier la dynamique d'alors. Il ressort de diverses études à notre disposition qu'au moins une trentaine de pays ont instauré ou réformé leurs réglementations bancaires durant les années $1930^{(6)}$. Les seules exceptions notables à cette vague réglementaire, parmi les places financières internationales, sont le Royaume-Uni, la France et les Pays-Bas. Une forte tendance à contrôler les activités bancaires est donc clairement identifiable. Les crises ont ébranlé la confiance dont bénéficiaient les principes d'autorégulation et de solidarité de place. Les régulations prudentielles instaurées ont bien souvent une visée préventive - amélioration de la transparence des bilans, détermination de ratios de solvabilité et de liquidité -, à laquelle s'ajoute parfois une réforme structurelle - spécialisation bancaire, séparation des activités de banque de dépôts et banque d'investissement ${ }^{(7)}$. Dans la foulée de la récente crise financière de 2007-2008, les commentaires faisant référence à la sagesse des

(5) Cf. par exemple Alfredo Gigliobianco, «Introduction. Regulatory Responses To Financial Crises. In Search of a Pattern», dans Alfredo Gigliobianco \& Gianni Toniolo, eds., Financial Market Regulation in the Wake of Financial Crises. The Historical Experience, Rome, Banca d'Italia Eurosistema, 2009, p. 9: «The crisis which started in 1929 really represents a watershed in the matter of regulatory responses. While the reaction to previous crises had been rather mild and scattered, in the mid-Thirties the banks, or the larger set of financial institutions, had to submit to comprehensive and forceful legislation almost everywhere».

(6) Selon des publications contemporaines, les pays suivants sont concernés: Danemark (avril 1930), Afrique du Sud (loi modifiée en mai 1930), Chine (mars 1931), Grèce (juin-juillet 1931), Brésil (loi modifiée en 1931 et 1934), Yougoslavie (janvier 1932), Estonie (mars 1932), Tchécoslovaquie (loi modifiée en avril 1932), Hongrie (juin 1932), Australie (loi modifiée en 1932), Autriche (août 1932 et avril 1933), Finlande (mars 1933), Bulgarie (mai 1933), Suède (loi modifiée en juin 1933, 1936-1937), République de Dantzig (août 1933), Nouvelle Zélande (1933), USA (juin 1933, août 1935), Roumanie (mai 1934), Canada (loi modifiée en juin 1934), Norvège (modifié juin 1934), Pologne (juin 1934), Suisse (novembre 1934), Allemagne (décembre 1934), Mexique (décembre 1934), Argentine (mars 1935), Belgique (juillet 1935), Chili (juillet-août 1935), Italie (mars 1936), Turquie (juin 1936), Costa Rica (1936), Uruguay (septembre 1936), Palestine (1936-1937), Espagne (août 1938). Cf. Paul GrANER, Der Geltungsbereich des Bankengesetzes, Zurich, Polygraphischer Verlag, 1937, p. 116-118; Johannes C.D. ZAHN, ed., Die Bankaufsichtsgesetze der Welt, Berlin-Leipzig, Walter de Gruyter \& Co., 1937; Eugène SMITs, Étude critique de l'intervention de l'Etat dans la politique de crédit des banques de dépôts, Bruxelles, Cools, 1940, p. 37-39.

(7) Cf. Hubert BONIN, «Crises et réglementation bancaire dans l'histoire européenne. Quelques rappels pour nourrir les débats actuels», dans Loïc GRARD \& Pascal KAUFMANN, eds., L'Europe des banques. Approches juridique et économique. Concurrence, réglementation, marché unique, Paris, Pedone, 2010, p. 81-96. 
principes de séparation des activités bancaires et en particulier à certaines mesures du Glass-Steagall Act américain de 1933 sont d'ailleurs légion ${ }^{(8)}$.

Parmi les pays ayant instauré un régime de surveillance bancaire dans les années 1930, l'on compte également deux petits États européens de nature comparable, la Suisse et la Belgique ${ }^{(9)}$. Les législations bancaires de ces deux small open economies ${ }^{(10)}$ sont encore relativement peu étudiées par les historiens. Sur le cas suisse, les travaux d'historiens sont encore rares. Mis à part dans la thèse de Hugo Bänziger sur le développement de la surveillance bancaire ${ }^{(11)}$, l'élaboration de la loi de 1934 n'a fait l'objet que de quelques études, souvent focalisées sur un aspect particulier ${ }^{(12)}$. Sur la Belgique, l'historiographie du contrôle bancaire se résume également à un nombre limité de contributions. À partir des travaux de Guy Vanthemsche sur la conception de la législation bancaire et plus généralement l'action économique de l'État belge dans les années $1930^{(13)}$, d'autres chercheurs ont abordé cette

(8) Cf. par exemple Christian NOYER, ed., Quel avenir pour la régulation financière?, Revue de la Stabilité Financière, Paris, Banque de France, 2009; Barry EICHENGReEn, «Macroeconomic and Financial Policies Before and After the Crisis», dans Maurice OBSTFELD, Dongchul CHO \& Andrew MASON, eds., Global Economic Crisis. Impacts, Transmission, Recovery, Cheltenham, Edward Elgar, 2012, p. 181-203.

(9) De dimensions géographique et démographique relativement proches (33.000 $\mathrm{km}^{2}$ pour 8,1 millions d'habitants en Belgique, $41.000 \mathrm{~km}^{2}$ pour 4,1 millions d'habitants en Suisse en 1930). Les PNB des deux pays s'élèvent à 66,5 milliards de FB courant en 1930, 9,95 milliards de CHF courant en 1930 (soit environ 68,86 milliards de FB). Sources: Brian R. Mitchell, International Historical Statistics: Europe 1750-2000, Basingstoke, Palgrave Macmillan, 2003, p. 3, 8, 908, 913. Pour la conversion: Banque nationale Suisse, Manuel statistique du marché financier suisse, Zurich, Schulthess, 1944, p. 46-49.

(10) Sur ce concept: Peter J. KATZENSTEIN, Small States in World Markets. Industrial Policy in Europe, Ithaca, Cornell University Press, 1985.

(11) Hugo BäNZIGER, Die Entwicklung der Bankenaufsicht in der Schweiz seit dem 19. Jahrhundert, Berne-Stuttgart, P. Haupt, 1986.

(12) Malik MAZBOuRi \& Janick Marina SchaufelbueHL, "A Legislator under Surveillance: The Creation and Implementation of Swiss Banking Legislation 1910-1934», dans European History Quarterly, à paraître; Thibaud GIDDEY, "Gendarme ou médecin des banques? Les premières années d'activité de la Commission fédérale des Banques (1935-1943)», dans Traverse. Revue suisse d'Histoire, 2012, n 3, p. 145-163; Philipp Müller, La Suisse en crise (1929-1936), Lausanne, Antipodes, 2010, p. 309-321; Marc PERRENOUd et al., La place financière et les banques suisses à l'époque du nationalsocialisme : les relations des grandes banques avec l'Allemagne (1931-1946), Zurich, 2002 (Chronos, 13), p. 107-122; Patrick HALBEISEN, «The Banking Crisis and its Implications for Swiss Banking Legislation in the 1930s », dans Manfred POHL, Teresa TORTELLA \& Herman VAN DER WeE, eds., A Century of Banking Consolidation in Europe, Aldershot, Ashgate, 2001, p. 102-120; Sébastien GueX, «The Origins of the Swiss Banking Secrecy Law and Its Repercussions for Swiss Federal Policy», dans Harvard Business History Review, t. 74, 2000, p. 237-266; Paul EHRSAM, «Die Bankenkrise der 30er Jahre in der Schweiz», dans Urs Zulauf, ed., 50 ans de surveillance fédérale des banques, Zurich, Schulthess Polygraphischer Verlag, 1985, p. 83-118.

(13) Guy Vanthemsche, "The Economic Action of the Belgian State During the Crisis of the 1930s», dans Herman VAN DER WeE \& Jan Blomme, eds., The Economic Development of Belgium since 1870, Cheltenham, Edward Elgar, 1997, p. 337-356; ID., «De politieke en economische context van de Belgische bankwetgevingen van 1934 en 1935 », dans Revue de la Banque - Bank en financiewezen, 1980, p. 31-50; ID., "L'élaboration de l'Arrêté royal sur le contrôle bancaire (1935)", dans Revue belge d'Histoire contemporaine, t. 11,1980 , p. $389-437$. 
importante étape de l'histoire économique belge, dans une perspective tantôt macroéconomique et diachronique ${ }^{(14)}$, tantôt juridique ${ }^{(15)}$, tantôt biographique ${ }^{(16)}$. Or, si une poignée de ces études mentionnent furtivement les éventuelles ressemblances de la loi bancaire belge avec son homologue suisse ${ }^{(17)}$, aucun chercheur n'a encore interrogé les liens de parenté entre les deux régimes de régulation, dans une perspective comparative. Relevons d'ailleurs d'emblée qu'un certain nombre d'observateurs ne mentionnent pas ou nient l'influence des potentielles sources d'inspiration étrangères, préférant insister sur l'originalité proprement belge des solutions envisagées. Ainsi, R.J. Lemoine, chef de cabinet du Ministre des travaux publics socialiste Henri de Man, écrit-il dans un commentaire sur la réglementation bancaire belge en cours d'élaboration que «l'apport de la législation étrangère dans la confection de cet arrêté est presque nul» ${ }^{(18)}$. Ce jugement peut être sérieusement nuancé. Pour aborder cette problématique, l'article procède comme suit: après avoir rapidement esquissé le contexte politique et économique dans lequel s'inscrivent les discussions qui mènent à l'adoption de l'arrêté royal $\mathrm{n}^{\circ} 185$ du 9 juillet 1935, l'analyse se concentrera en premier lieu sur les divers indices qui témoignent d'une inspiration de la loi suisse durant le processus d'élaboration législative. En second lieu, le point de vue se déplacera sur le résultat auquel ces influences ont abouti: la comparaison entre d'une part les lois bancaires belge et suisse, et d'autre part les instances de surveillance respectives créées pour l'occasion.

\section{Le développement du contrôle bancaire belge et son contexte (1931-1935)}

La profonde crise économique mondiale initiée en 1929 affecte gravement l'économie belge, fortement dépendante des marchés extérieurs. La chute de la demande internationale, combinée au développement de mesures

(14) Jean-Louis MOREAU, «Contrôler sans nationaliser. Le contrôle bancaire en Belgique de 1935 à 1974», dans Vincent DuJARDIN et al., eds., La crise économique et financière de 2008-2009. L'entrée dans le 21e siècle?, Bruxelles, Peter Lang, 2010, p. 107-120; Ivo MAES \& Erik BUYST, «Financial Crisis and Regulation. An Overview of the Belgian Experience», dans A. Giglobianco \& G. TONIOLO, eds., Financial Market Regulation, op. cit., p. 95-117; Isabelle CASSIERS et al., «Les banques belges face à l'État : une rétrospective (1935-1993)», dans Revue d'Économie financière, t. 48, 1998, p. 125-155.

(15) Dimitiri YeRnault, L'État et la propriété. Le droit public économique par son histoire (1830-2012), Bruxelles, Bruylant, 2013, p. 521-529. Cf. également André BRUYNEEL, «The Belgian 'Commission bancaire': Functions and Methods », dans Journal of Comparative Corporate Law and Securities Regulation, t. 1, 1978, n 2, p. 187-209.

(16) Ginette Kurgan-Van Hentenryk, Max-Léo Gérard. Un ingénieur dans la cité (1879-1955), Bruxelles, Éditions de l'Université de Bruxelles, 2010, en part. p. 147-192.

(17) G. VANTHEMSCHE, «L'élaboration de l'arrêté», op. cit., p. 432; G. VANTHEMSCHE, «De politieke», op. cit., p. 48; I. MAES \& E. BUYST, «Financial Crisis and Regulation», op. cit., p. 104.

(18) IEV, Fonds Lemoine, I.B.3/13, document intitulé «Le contrôle des banques», 29 juin 1935. Dans son article sur le contexte économique, politique et idéologique des réformes bancaires de 1934-1935, Guy Vanthemsche, bien qu'il reconnaisse certaines ressemblances avec la loi suisse, relativise l'importance des exemples étrangers: «Zo belangrijke hervormingen als die van de KB's $\mathrm{n}^{\mathrm{r}} 2$ en 185 worden niet ingevoerd op basis van zekere theoretische principes of buitenlandse voorbeelden». G. VANTHEMSCHE, «De politieke», op. cit., p. 46. 
protectionnistes, provoque un effondrement des exportations ${ }^{(19)}$. La position concurrentielle de la Belgique s'empire encore lors de la dévaluation de la Livre Sterling en septembre 1931, que le gouvernement belge décide de ne pas suivre. Au contraire, comme en Suisse, la réaction gouvernementale à la crise prend la forme d'une politique déflationniste, visant à rétablir la compétitivité des produits belges par le biais d'une baisse des prix, et partant, d'une réduction des coûts de production. Le système bancaire belge, caractérisé par le rôle prépondérant des grandes banques mixtes, telles que la Société générale de Belgique et la Banque de Bruxelles, détenant d'importantes participations industrielles, est sévèrement touché par la récession économique. La dépréciation des titres détenus par les banques, les pertes importantes subies suite à l'insolvabilité d'entreprises, les retraits de dépôts et l'illiquidité qui s'ensuivent sont autant des facteurs aggravant la crise financière ${ }^{(20)}$. Malgré des tentatives d'intervention de la Banque nationale de Belgique, la liquidité du système bancaire s'érode davantage. Les dépôts bancaires passent de 4,774 à 3,636 millions de francs-or entre 1930 et $1934^{(21)}$. Après une première vague de faillites bancaires en 1931-1932, la Banque Belge du Travail et l'Algemeene Bankvereeniging - banques populaires de taille moyenne patronnées respectivement par le Parti ouvrier belge et le Boerenbond catholique, font face à des difficultés en 1934. Les liens politiques de ces établissements financiers provoquent l'irruption dans le débat politique des questions relatives à leur gestion et à leur sauvetage ${ }^{(22)}$. Ces événements vont précipiter l'adoption de mesures gouvernementales en août 1934. Un premier arrêté autorise les banques à se servir de la Société nationale de Crédit à l'Industrie (23) pour échanger des créances non liquides contre des obligations à garantie étatique. Simultanément, par un second arrêté, le gouvernement catholiquelibéral dirigé par Charles de Broqueville (1860-1940) instaure la scission des banques mixtes. Celles-ci se séparent donc en deux entités juridiques formellement distinctes, la banque de dépôt d'une part et la société finan-

(19) En 1934, les exportations ne représentaient plus que 42,6\% de leur niveau de 1929. Herman VAN DER WEE \& Karel TAVERNIER, La Banque nationale de Belgique et l'histoire monétaire entre les deux guerres mondiales, Bruxelles, Banque nationale de Belgique, 1975, p. 255. I. MAES \& E. BUYST, «Financial Crisis and Regulation», op. cit., p. 100.

(20) Erik BuYst et al., La Banque nationale de Belgique, du franc belge à l'euro. Un siècle et demi d'histoire, Bruxelles, Racine, 2005, p. 133; I. CASSIERS, «Les banques belges», op. cit., p. 127-128.

(21) Guy VANTHEMSCHE, «State, Banks and Industry in Belgium and The Netherlands, 1919-1939», dans Harold JAMES, Håkan LINDGREN \& Alice TEICHOVA, eds., The Role of Banks in the Interwar Economy, Cambridge, Cambridge University Press, 1991, p. 104121, ici p. 110.

(22) Ginette KuRgan-VAn HentenRYK, «Les banques privées belges de 1920 à nos jours », dans Maurice LÉVY-LEBOYER, ed., Les banques en Europe de l'Ouest de 1920 à nos jours, Paris, Comité pour l'Histoire économique et financière de la France, 1995, p. 139-152, ici p. 142; André Mommen, The Belgian Economy in the Twentieth Century, London-New York, Routledge, 1994, p. 22-23.

(23) La Société nationale de Crédit à l'Industrie est une institution parastatale créée en 1919 avec pour but le financement d'entreprises à moyen terme par le biais d'émissions d'obligations à garantie étatique. H. VAN DER WEE \& K. TAVERNIER, La Banque nationale de Belgique, op. cit., 1975, p. 67-68. 
cière à participations industrielles d'autre part, les deux activités étant désormais incompatibles. Une conjonction de facteurs, selon Guy Vanthemsche, est à l'origine de cette réforme ${ }^{(24)}$. Premièrement, il faut souligner que cette mesure n'est pas prise à l'encontre des intérêts des banques concernées, mais a au contraire été suggérée par les plus importants dirigeants bancaires ${ }^{(25)}$. Dans le discours officiel, une meilleure protection de l'épargne et la restauration de la confiance du public dans les institutions financières sont invoquées pour justifier la réforme. Du point de vue du gouvernement, l'opération constitue une contrepartie au mécanisme de refinancement des banques par la Société nationale de Crédit à l'Industrie mis en place parallèlement. Du point de vue des banques, les motivations sont également diverses. D'une part, la scission des banques mixtes confirme une tendance initiée par les banques elles-mêmes depuis les années 1920 vers une plus grande spécialisation dans la gestion de leurs portefeuilles-actions. D'autre part, la réforme permet aux grandes banques de dissimuler, dans la refonte des bilans, des amortissements devenus nécessaires. Autre avantage immédiat, la réorganisation et les restructurations internes donnent lieu à des exonérations fiscales et une réduction des frais généraux. Dans l'ensemble, les arrêtés du 22 août 1934 sur la scission ne sont pas dirigés contre les intérêts des banques; ce que confirme le fait qu'aucun contrôle de l'application des mesures n'est prévu et que l'opération restera longtemps une scission de façade ${ }^{(26)}$.

Mais les réformes d'août 1934 ne suffisent pas à enrayer la crise financière. La fuite des capitaux, les attaques spéculatives contre le franc belge, et l'échec de la politique déflationniste rendent une réaction gouvernementale indispensable pendant l'hiver 1934-1935. Fin mars 1935, Paul Van Zeeland (1893-1973) forme un nouveau gouvernement d'union nationale et impose une dévaluation du franc dont la valeur est réduite de $28 \%$. Ses effets bénéfiques se font rapidement sentir: un afflux de capitaux soulage la liquidité des banques, tandis que le redressement des exportations permet à l'économie belge de profiter de la reprise conjoncturelle internationale ${ }^{(27)}$. Le gouvernement Van Zeeland tripartite (six ministres catholiques, cinq socialistes, quatre libéraux) est un mélange de jeunes éléments réformateurs ${ }^{(28)}$, mais intègre également des membres conservateurs, comme le libéral Max-Léo Gérard (1879-1955), qui se voit confier le portefeuille des finances ${ }^{(29)}$.

(24) G. VAnthemsche, «De politieke», op. cit., p. 37-40; ID., «State, Banks and Industry», op. cit., p. 111-112.

(25) En particulier Émile Francqui (1863-1935), gouverneur de la Société générale de Belgique.

(26) Guy VAnTHemsche, «La Banque de 1934 à nos jours», dans Herman VAN DER WeE, ed., La Générale de Banque. 1822-1997, Bruxelles, Racine, 1997, p. 287-526, ici p. 311-319.

(27) E. Buyst, La Banque nationale de Belgique, op. cit., p. 136.

(28) Dumoulin insiste sur le tournant générationnel que représente «l'expérience Van Zeeland». Michel DumOuLIN, «Les interventions de crise de l'État belge durant la grande dépression», dans V. DuJARDin, ed., La crise économique, op. cit., p. 95-106. Cf. aussi Isabelle CASSIERS, Croissance, crise et régulation en économie ouverte. La Belgique entre les deux guerres, Bruxelles, De Boeck, 1989, p. 179.

(29) Sur Max-Léo Gérard et son rôle dans le gouvernement Van Zeeland: Ginette KURGAN-VAN HENTENRYK, «Max-Léo Gérard, un ministre des Finances méconnu», dans 
Le programme gouvernemental, fruit d'une longue négociation préalable à la formation du cabinet et présenté le 29 mars 1935 au Parlement, inclut déjà la mise en place d'un "contrôle sur les banques». Sans entrer dans les détails de ce projet, relevons que pour veiller sur la politique du crédit, la déclaration gouvernementale prévoit la création d'«un organisme qui agira au nom et pour le compte de la puissance publique; il ne sera pas une administration dépendante d'un ministère, mais il pourrait être la Banque nationale de Belgique ou un organisme géré par elle»( ${ }^{(30)}$. Cette formule laconique et floue est sans doute représentative des tensions entre les diverses tendances politiques au sein du gouvernement. Elle est également significative dans l'histoire de la conception de la Commission bancaire. En outre, cette première déclaration gouvernementale constitue déjà une forme de compromis politique; les projets initiaux du Parti ouvrier belge dans le Plan du Travail de décembre 1933, qui prévoyaient la nationalisation et l'orientation du crédit par l'État, ont été abandonnés lors de la formation du gouvernement en mars $1935^{(31)}$. Il faut encore signaler que le nouveau cabinet se voit octroyer des "pouvoirs spéciaux» en vue de la réalisation de son programme financier, procédure qui lui permet d'élaborer la législation bancaire en dehors des débats parlementaires et publics.

L'élaboration de l'arrêté royal $n^{\circ} 185$ du 9 juillet 1935 sur le contrôle bancaire a fait l'objet d'un examen détaillé et précis ${ }^{(32)}$. L'analyse de Guy Vanthemsche insiste sur les deux tendances essentielles qui se confrontent, au sein même du gouvernement, dans le processus de décision. D'une part, des premiers projets de loi sont rédigés au sein de l'Office de Redressement économique (OREC), un service interministériel chargé de coordonner la politique conjoncturelle et d'étudier les réformes de structure ${ }^{(33)}$. Ces premiers projets prévoient notamment la création d'un «Office central des Banques», placé sous l'influence de l'OREC. Cet organisme serait composé d'une «section économique qui étudie toutes questions relatives au crédit et au marché des capitaux et d'une section de vérification comptable et de surveillance qui veille à l'application des lois et arrêtés qui régissent l'activité des banques et établissements financiers» (34). D'autre part, parallèlement, le ministre des Finances Max-Léo Gérard et son chef de cabinet, le jeune économiste Eugène de Barsy (1906-1985), entament également le travail d'élaboration d'un contrôle bancaire. Le ministère des Finances rédige des projets de loi concurrents de ceux proposés par l'OREC. Le processus de remaniements et de discussion des textes législatifs du ministère des Finances est marqué

Synthèses de droit bancaire et financier. Liber Amicorum André Bruyneel, Bruxelles, Bruylant, 2008, p. 667-680 ; EAD., Max-Léo Gérard, op. cit., en part. p. 147-192.

(30) Annales parlementaires, Chambre, 29 mars 1935, p. 689, cité dans Commission bancaire 1935-1960, Bruxelles, Commission bancaire, 1960, p. 40.

(31) G. VANTHEMSCHE, «L'élaboration de l'arrêté», op. cit., p. 400.

(32) Ibid.

(33) Sur l'OREC et ses échecs: Guy VANTHEMSCHE, «De mislukking van een vernieuwde economische politiek in België voor de Tweede Wereldoorlog: de OREC (Office de Redressement économique) van 1935 tot 1938 », dans Belgisch Tijdschrift voor Nieuwste Geschiedenis, $\mathrm{n}^{\circ} 2-3,1982$, p. 339-389.

(34) ABNB, dossier O303/4, Projet Lemoine ${ }^{\circ} 2,5$ juin 1935, art. 8. 
par l'influence non-négligeable des milieux bancaires ${ }^{(35)}$. En l'espace de six semaines, plus d'une dizaine de consultations ont lieu entre Gérard et divers banquiers de premier plan ${ }^{(36)}$. Ces derniers ont même constitué un comité chargé de négocier avec le ministère des finances la portée du contrôle bancaire: présidé par le gouverneur de la Société générale de Belgique, Alexandre Galopin (1879-1944), ce groupement informel intègre d'autres établissements financiers d'importance: la Banque de Bruxelles, la Kredietbank, le Crédit anversois et la Banque d'Anvers y sont notamment représentés ${ }^{(37)}$.

Le Premier ministre Paul Van Zeeland joue donc un rôle d'arbitre entre deux pôles opposés: celui de l'OREC, dont les projets interventionnistes sont soutenus par le ministre des Travaux publics, le socialiste Henri de Man (1885-1953), et celui du ministre des Finances Max-Léo Gérard, faisant la part belle aux opinions des milieux intéressés. Selon Vanthemsche, cet arbitrage a largement penché en faveur des législateurs du ministère des Finances: «La version libérale du contrôle bancaire triompha largement» ${ }^{(38)}$. À travers les différentes refontes des projets et l'intervention du ministre des Finances, l'OREC perd la maitrise de l'élaboration de la législation. Les mesures de contrôle initialement proposées sont progressivement édulcorées, avant qu'un autre avant-projet, cette fois rédigé par de Barsy, ne devienne le texte de référence. Avant de nous pencher sur le régime de surveillance bancaire auquel ce processus a abouti, examinons de plus près cette phase d'élaboration sous l'angle de l'influence des législations étrangères et en particulier de la loi sur les banques suisse.

\section{Les inspirations étrangères de l'arrêté royal $n^{\circ} 185$ sur le contrôle des banques}

De nombreux contacts, comme en témoignent les archives, existent entre les autorités monétaires suisses et belges. Rien de plus normal, somme toute, que de fréquents contacts entre deux banques centrales faisant partie du Bloc-or. Il est en revanche intéressant de souligner que ces échanges ne se limitent pas au strict domaine de la politique monétaire. Ainsi, la correspondance entre Louis Franck (1868-1937, gouverneur de la BNB) et Gottlieb Bachmann (1874-1947, président de la Banque nationale suisse) contient plus d'une dizaine de lettres entre 1933 et 1934, qui concernent les mesures prises en Suisse contre la crise financière (création de la Caisse de prêts de la Confédération, développement de la législation bancaire suisse) ${ }^{(39)}$. Il ne semble pourtant pas que ces fréquentes transmissions d'informations

(35) G. VAnthemsche, «L'élaboration de l'arrêté», op. cit., p. 412-414.

(36) AGR, Papiers Max-Léo Gérard, dossier 71, note du 12 octobre 1935.

(37) AGR, Papier Max-Léo Gérard, dossier 31, lettre de Max-Léo Gérard à Paul Van Zeeland, 17 avril 1935. Cité dans G. VANTHEMSCHE, «L'élaboration de l'arrêté», op. cit., p. 410. Voir aussi: IEV, Fonds Lemoine, I.B.1/11, note R. Lemoine à Henri de Man, 5 avril 1935.

(38) G. VANThemsche, «L'élaboration de l'arrêté», op. cit., p. 431.

(39) ABNB, Dossier A 277/3, onze lettres échangées entre janvier 1933 et juin 1934. 
réciproques aient eu un impact sur le développement du contrôle bancaire belge. Comme le souligne Ginette Kurgan-van Hentenryk, les dirigeants de la $\mathrm{BNB}$, à cause de leur opposition à la récente dévaluation, sont mis de côté durant le processus d'élaboration de l'arrêté royal $\mathrm{n}^{\circ} 185^{(40)}$. Malgré leurs contacts avec les banquiers suisses, il est donc peu pertinent de considérer les dirigeants de la Banque nationale de Belgique comme les principaux promoteurs du modèle suisse ${ }^{(41)}$.

Qu'en est-il dans les premiers projets de loi issus de l'OREC, ce «cheval de Troie planiste » ${ }^{(42)}$ ? Les projets de loi rédigés par Lemoine n'ont que très peu de points communs avec la réglementation helvétique. Comme indiqué plus haut, pas de trace dans ces projets d'une «Commission bancaire»; on y trouve plutôt un «Office central des Banques», aux compétences assez larges, tel qu'en témoigne la disposition qui l'autorise à «prescrire toute mesure propre à renforcer la situation des banques de dépôt et à contribuer ainsi à la protection de l'épargne» ${ }^{(43)}$. Parmi le large éventail des mesures à disposition de l'Office, l'on compte notamment la prescription de réserves extraordinaires, la suspension de la distribution d'un dividende, ou l'obligation d'investir la moitié des réserves bancaires en fonds publics belges. De plus, si le projet prévoit certes un rôle important aux «comptables-assermentés», il confie aussi à la Banque nationale un rôle d'inspecteur des banques, capable de mener des inspections personnelles selon certaines conditions. Selon Albert Halasi (1887-1965), dont les textes produits au Bureau d'Études sociales du Parti ouvrier belge ont servi de canevas aux projets Lemoine, ce sont plutôt les législations allemande et américaine, et non pas suisse, dont se sont inspirés les socialistes belges ${ }^{(44)}$.

Au ministère des Finances, l'équipe Gérard-de Barsy n'est pas non plus imperméable aux exemples de lois étrangères existantes. Dans une conférence donnée en 1945, alors qu'il n'est plus ministre des Finances mais président de la Brufina - la seconde plus grande société financière du pays -, Max-Léo Gérard revient sur les circonstances de la préparation de la loi bancaire. Il se souvient des longues soirées en compagnie d'Eugène de Barsy consacrée à l'étude des textes étrangers, en particulier «les textes les plus récents de la réglementation des banques en Allemagne et en Suisse» ${ }^{(45)}$.

(40) Ginette Kurgan-VAn HentenryK, «The Banque nationale de Belgique and the Belgian Economy in the Twentieth Century», dans Luigi DE ROSA, ed., International Banking and Financial Systems. Evolution and Stability, Aldershot, Ashgate, 2003, p. 117144, ici p. 124 ; H. VAN DER WEE \& K. TAVERNIER, La Banque nationale de Belgique, op. cit., p. 364. Soulignons que la BNB est représentée indirectement par les économistes R.J. Lemoine et L. Dupriez, tous deux au service de la banque centrale avant d'être nommés à l'OREC.

(41) Contrairement à l'interprétation proposée par Thomas Daelmans, dans son récent mémoire consacré à la supervision bancaire belge dans les années 1930: Thomas DAELMANS, Een crisis in de crisis. Een zoektocht naar bankencontrole in België tijdens de jaren dertig, Katolieke Universiteit Leuven, 2013, p. 31.

(42) G. VANTHEMSCHE, «L'élaboration de l'arrêté», op. cit., p. 408.

(43) IEV, Fonds Lemoine, I.B.1/3, Projet Lemoine n 3, 7 juin 1935.

(44) IEV, Fonds Lemoine, I.B.1/1, lettre d'Albert Halasi à Henri de Man, 4 juin 1935.

(45) Max-Léo GÉRARD, «À propos d'un anniversaire. Naissance et développement de l'arrêté $\mathrm{n}^{\circ} 185$ du 9 juillet 1935, sur le contrôle des banques et le régime des émissions de titres et valeurs», dans Centre d'Études bancaires, t. 19, 1945, p. 17-36, p. 23. 
Ces souvenirs sont corroborés par les documents d'archives. Le 4 juin 1935, Gérard écrit au Premier ministre Paul Van Zeeland les lignes suivantes: «En ce qui concerne le régime des banques, je serai saisi prochainement par M. GALOPIN, qui a pris en mains l'achèvement de cette étude, d'un projet exprimant les vues des banquiers et qui serait essentiellement basé sur le système suisse» ${ }^{(46)}$. L'impact de ce projet «Galopin», basé sur le système suisse ne tarde pas à se faire sentir dans les refontes des textes à l'échelon gouvernemental. Les 15 et 17 juin 1935 ont lieu deux réunions entre Paul Van Zeeland et les économistes de l'OREC, Robert J. Lemoine (1897-1938) et Léon-H. Dupriez (1901-1986), dont le procès-verbal confidentiel est très éloquent ${ }^{(47)}$. Plusieurs indices permettent d'affirmer que le projet est considérablement remanié à partir de ce moment, en prenant en considération le modèle suisse. De plus, l'ensemble de ces modifications est à mettre au crédit du Premier ministre. Dès les remarques préliminaires, Paul Van Zeeland affirme qu'il «conçoit comme entrant dans le statut légal des banques: $1^{\circ}$ ) la fixation d'un capital minimum, l'établissement d'un rapport entre le capital propre et les dépôts, entre les disponibilités liquides et les placements, $2^{\circ}$ ) le fonctionnement des banques, la responsabilité des administrateurs, l'institution de 'chartered accountant'». L'ensemble de ces dispositions se révèle être des mesures contenues dans la réglementation bancaire helvétique. Tant les ratios de liquidité et de solvabilité, les mesures renforçant la responsabilité des dirigeants bancaires, que l'officialisation du rôle des auditeurs font partie des fondements essentiels de la loi suisse ${ }^{(48)}$. Certes elle n'en a pas le monopole, mais leur intégration inopinée dans les projets de loi belges demeure frappante.

Cette inspiration n'apparaît d'ailleurs pas uniquement dans les nouveaux principes qui sont incorporés, mais également dans les vocables utilisés pour les désigner. Ainsi, Paul Van Zeeland insiste-t-il pour que le terme de «reviseur», identique à l'expression du droit suisse, soit employé au lieu de celui de «comptable assermenté» ${ }^{(49)}$. Les expressions «revision» et «reviseur» apparaissent ainsi pour la première fois dans le droit belge avec l'arrêté royal $\mathrm{n}^{\circ} 185$, avant d'être étendu en 1953 à l'ensemble de l'économie ${ }^{(50)}$. De plus, l'Office central des Banques, mentionné plus haut et encore présent dans les projets Lemoine, cède sa place à une «Commission bancaire», dans laquelle l'OREC n'a plus aucun rôle à jouer, et qui serait «une émanation, pour partie,

(46) AGR, Papiers Max-Léo Gérard, dossier 31, lettre de Max-Léo Gérard à Paul Van Zeeland, 4 juin 1935.

(47) ABNB, O 303/4, Procès-verbal confidentiel de la réunion tenue au cabinet du Premier ministre, le 15 juin1935, sous la présidence de M. Van Zeeland, 14 pages. IEV, Fonds Lemoine, I.B.3/6, procès-verbal confidentiel de la réunion tenue au cabinet du $1^{\text {er }}$ Ministre le 17 juin 1935, 4 pages.

(48) Il s'agit respectivement des articles 4 (fonds propres et liquidité), 39-45 (responsabilité) et 18-22 (contrôle et revision) de la loi fédérale sur les banques et les caisses d'épargne du 8 novembre 1934.

(49) ABNB, O 303/4, Procès-verbal confidentiel de la réunion tenue au cabinet du Premier ministre, le 15 juin 1935, sous la présidence de M. Van Zeeland, p. 13.

(50) Daniel Goosens, "Les professions de reviseur de banques et de reviseur d'entreprises », dans Reflets et Perspectives de la Vie économique, $\mathrm{n}^{\circ}$ 1, 1971, p. 3-16, p. 11; Michel CHARVET, La crise du système bancaire belge et sa réorganisation récente, Paris, Sirey, 1936, p. 166, n. 1. 
des milieux intéressés, avec prédominance de l'intérêt public». R. Lemoine ne cache pas sa déception sur les moyens d'action de ce nouvel organisme: «ou bien elle aura des pouvoirs arbitraires et exorbitants, ou bien elle n'en aura aucun et c'est l'éventualité la plus probable» ${ }^{(51)}$. Quant à la composition de cette autorité de surveillance, il est décidé «de déterminer les qualités des membres de la Commission bancaire, en s'inspirant de la loi suisse » ${ }^{(52)}$. Cela n'est qu'en partie vrai, puisque la loi belge sera plus précise sur le choix des membres de la Commission bancaire. Elle prescrit notamment que deux d'entre eux seront choisis sur une liste fournie par les représentants des banques, et deux autres sur une liste présentée conjointement par la BNB et l'Institut de Réescompte et de Garantie ${ }^{(53)}$. Ces dispositions limitent donc d'une part la marge de manœuvre du gouvernement dans la composition de la Commission bancaire, et formalise d'autre part dans la loi le rôle des industries régulées dans le choix des régulateurs.

L'intervention de Paul Van Zeeland, inspirée des vœux des milieux bancaires belges basés sur le système suisse, transforme entièrement le type de régime de contrôle bancaire. Les réformes de grande envergure de l'OREC, qui prévoyaient la création d'une inspection des banques par une autorité publique (comparable au système américain ou aux inspectorats scandinaves), sont remplacées par un système de surveillance indirect, qui confie le contrôle primaire à des reviseurs privés, choisis par les banques, et dans lequel l'émanation de l'État (la Commission bancaire) n'a qu'un pouvoir limité. L'influence du modèle suisse, dont les banquiers belges se sont inspirés, par l'intermédiaire de Max-Léo Gérard et de Paul Van Zeeland, modifie foncièrement les ébauches de loi, très précisément entre le 4 juin et le 17 juin 1935 .

Un examen attentif des divers projets de loi produits par les législateurs belges révèle qu'à partir des deux réunions de mi-juin 1935 évoquées ci-dessus, et malgré de nombreux remaniements le texte qui deviendra l'arrêté royal du 9 juillet 1935 ne changera pas fondamentalement. Parmi les derniers changements opérés, il faut relever l'insistance avec laquelle les milieux bancaires demandent l'exclusion des sociétés financières - les holdings détenant seules les portefeuilles-titres industriels depuis la scission d'août 1934 du champ d'application de la réglementation ${ }^{(54)}$. Contrairement à ce que l'on pourrait croire, les banques n'ont pas cherché à remettre en question en 1935 les mesures de séparation bancaire prises en 1934. Elles se sont au contraire

(51) IEV, Fonds Lemoine, I.B.3/6, procès-verbal confidentiel de la réunion tenue au cabinet du $1^{\mathrm{er}}$ ministre le 17 juin 1935.

(52) Ibid.

(53) L'Institut de Réescompte et de Garantie, une autre œuvre du tandem Gérardde Barsy, est une institution spéciale créée le 15 juin 1935. Son but est de réescompter les actifs des banques privées à moyen ou à long terme, actifs que la BNB ne pouvait réescompter en raison de ses limites statutaires. Cet organisme concurrent de la BNB pouvait émettre des bons de caisse sous garantie de l'État pour financer ses opérations. Cf. H. VAN DER WEE \& K. TAVERNIER, La Banque nationale de Belgique, op. cit., p. 299-301.

(54) Cf. ABNB, O303/1, «Note sur le 'Projet de contrôle des banques'», non signé, daté à la main du 19 juin 1935. Voir aussi AGR, Papiers MLG, n 31, lettre de Max-Léo Gérard à Paul Van Zeeland, 26 juin 1935: Gérard y rapporte une conversation avec Galopin, qui s'oppose à la réglementation de tout autres institutions financières que les banques de dépôts. 
servies de ces dispositions pour justifier l'exclusion des sociétés financières du cadre général de la surveillance bancaire. Après une dernière phase chaotique, qui verra le Parti ouvrier belge (POB) imposer à la dernière minute une disposition qui autorise la Commission bancaire à fixer des taux d'intérêts maximaux ${ }^{(55)}$, l'arrêté royal sur le contrôle des banques et le régime des émissions de titres et valeurs est signé par le roi Léopold III le 9 juillet 1935 et entre en vigueur le $1^{\text {er }}$ septembre 1935.

\section{«Deux législations sœurs»}

Le processus d'élaboration de cet arrêté indique donc nettement que l'exemple suisse a joué un rôle dans la refonte des projets de loi belges. Qu'en est-il de la réglementation finale? Une comparaison, sur quelques points-clés, des deux réglementations permet de mettre en avant un certain nombre de similitudes et de différences. Il est premièrement remarquable que les deux textes législatifs suivent la même logique dans l'ordre d'exposition des articles. Certes l'agencement du droit, qu'il soit belge ou suisse, respecte souvent une même logique (définition de l'objet au début, sanctions pénales et dispositions transitoires à la fin). Il n'en reste pas moins frappant que les articles intermédiaires sont présentés dans le même ordre.

Les dissemblances entre les deux réglementations sont malgré tout relativement nombreuses. La loi belge ne contient pas de dispositions sur l'organisation interne des banques (délimitation du champ d'activité dans les statuts, distinction entre organes de direction et organes de surveillance), ni sur les mesures de moratoires prévues pour empêcher les runs bancaires et crises de liquidité, comme la prorogation des échéances ou le sursis bancaire. En outre, relevons qu'il n'existe dans la réglementation belge aucune disposition comparable au fameux article 47 de la loi bancaire suisse, qui protège le secret professionnel des banquiers dont la violation devient passible de sanctions de droit pénal ${ }^{(56)}$. En Belgique, seuls les reviseurs et les membres de la Commission bancaire sont soumis à des règles de confidentialité renforcée. Il est de plus précisé que l'autorité de surveillance «ne connaît pas de questions d'ordre fiscal» ${ }^{(57)}$. À l'inverse, la législation suisse ne prévoit pas de mesures

(55) De l'aveu même de président de la Commission bancaire, Georges Janssen, cette mesure nécessite de réunir tant de conditions qu'elle est inapplicable: «Si jamais quelqu'un parvient à mettre ce texte en vigueur, c'est qu'il aura exercé sur les autres une séduction personnelle particulièrement remarquable». Conférence devant le Jeune Barreau de Bruxelles, 23 janvier 1936.

(56) Sur le secret bancaire suisse et son renforcement dans la loi bancaire de 1934 : S. GUEX, «The Origins», op. cit.

(57) «Arrêté royal $n^{\circ} 185$ du 9 juillet 1935 sur le contrôle des banques et le régime des émissions de titres et valeurs », dans Moniteur belge, 10 juillet 1935, art. 24, 38 et 40. L'ajout de la petite phrase qui exclut les questions fiscales des compétences de la Commission bancaire est l'œuvre du Premier ministre Paul Van Zeeland, félicité à ce sujet par Max-Léo Gérard. Cf. AGR, Papiers MLG, n 31, lettre de Max-Léo Gérard à Paul Van Zeeland, 29 juin 1935. Sans suprise, cette clause qui exclut la fraude fiscale du droit de regard de la Commission bancaire n'est pas du goût des socialistes belges. Cf. Albert HALASI, «Le contrôle des banques», dans Le Mouvement syndical belge, 20 août 1935, $\mathrm{n}^{\circ} 8$, p. $168-171$. 
similaires à celles sur l'interdiction de détenir des participations dans des sociétés commerciales, sur les incompatibilités des fonctions dirigeantes dans les sociétés commerciales, sur la publicité financière, sur les fusions bancaires, ou encore sur les émissions de titres et valeurs. La différence la plus notable à long terme reste bien dans le champ d'application des lois et les réformes structurelles qu'elles impliquent. La législation suisse n'impose aucun changement dans les activités des institutions financières, elle consacre ainsi le modèle de la grande banque universelle. En Belgique, les réformes de 1934-1935 marquent une plus grande rupture dans l'évolution des établissements financiers. Sous l'impulsion des grandes banques, la séparation entre activité de banques de dépôts et de holdings industriels est introduite. Cette scission signifie également que les opérations des sociétés financières échappent complètement au contrôle mis en place en 1935.

$\mathrm{Au}$ rang des similitudes entre les deux systèmes de surveillance, il faut relever premièrement la disposition qui réserve la dénomination des termes «banque» et «banquier» aux établissements soumis à la loi ${ }^{(58)}$. Deuxièmement, les deux lois prévoient l'institution de rapports minimaux entre les fonds propres et les engagements (ratio de solvabilité) et entre les actifs aisément mobilisables et les engagements à court terme (ratio de liquidité). Il ne s'agit pas d'une mesure exceptionnelle parmi les diverses législations bancaires du monde ${ }^{(59)}$, mais à nouveau la formulation quasiment identique des dispositions est étonnante ${ }^{(60)}$. Troisièmement, les deux lois imposent aux banques, selon des modalités différentes ${ }^{(61)}$, la communication régulière de bilans comptables. Il s'agit là d'une mesure répandue. En revanche, il est intéressant de noter que l'établissement du schéma de bilan type par la Commission bancaire en 1937 s'est fait sur le modèle du schéma suisse $^{(62)}$. Quatrième élément inspiré du système suisse, la création d'un nouvel organisme de surveillance. La Commission bancaire belge, comme

(58) M. Charvet, La crise du système, op. cit., p. 159. De même, la loi belge, reprenant une disposition de la loi suisse, interdit la constitution de nouvelles banques de dépôts sous la forme de sociétés coopératives, le capital d'une société coopérative constituant une médiocre garantie. Franz DE VOGHEL, Contrôle des banques. Législations récentes, Gembloux, J. Duculot, 1936, p. 152.

(59) J. ZAHN, Die Bankaufsichtsgesetze, op. cit., p. XXII-XXIII; Arthur Meredith ALLEN et al., Commercial Banking Legislation and Control, Londres, Macmillan, 1938, p. 1423. La progressivité des taux prévus en Suisse (plus les engagements à court terme sont importants, plus le taux de couverture est élevé) semble en revanche être une innovation singulière. Cf. Fr. DE VoGHEL, Contrôle des banques, op. cit., p. 57-58.

(60) Cf. Franz DE Voghel, "La législation bancaire suisse», dans Centre d'Études bancaires, t. 2, 1938, p. 5-27, ici p. 8: «Les dispositions de la législation suisse relatives à la liquidité des banques ont manifestement inspiré les auteurs de la loi belge»; voir aussi ID., Contrôle des banques, op. cit., p. 143 et 155.

(61) En Suisse, la communication se fait sur base annuelle, semestrielle ou trimestrielle selon la taille des banques. Les bilans sont en revanche accessibles au public. En Belgique, les bilans et comptes pertes et profits sont communiqués annuellement à la Banque nationale. À cela s'ajoute la transmission mensuelle des situations - un état synthétique de la situation active et passive - toujours uniquement à destination de la Banque nationale de Belgique, qui, elle, publie régulièrement une situation globale des banques.

(62) M. Charvet, La crise du système, op. cit., p. 172; Fr. De Voghel, «La législation bancaire suisse», op. cit., p. 12. 
la Commission fédérale des banques, est composée en partie d'anciens banquiers, elle dispose d'une grande autonomie, à la fois vis-à-vis de la banque centrale et de l'administration gouvernementale ${ }^{(63)}$. Avant de revenir plus loin sur les raisons qui ont motivé ce choix institutionnel, relevons simplement qu'à l'échelle mondiale, la constitution d'une telle commission indépendante n'est en 1935 pas le modèle le plus répandu. De véritables inspectorats, étroitement liés au ministère des Finances, existent par exemple à ce moment-là en Finlande, Suède, Danemark, Norvège, au Japon et au Canada. Ailleurs, en Allemagne, en Italie ou encore en Argentine, les institutions de contrôle sont placées sous l'autorité de la banque centrale ${ }^{(64)}$. Enfin, la cinquième composante de la régulation bancaire belge inspirée de l'exemple suisse est celle du contrôle externe par des reviseurs ${ }^{(65)}$. Le système revisoral ainsi mis en place fait reposer le contrôle comptable primaire sur un corps de reviseurs privés, assermentés par l'autorité de surveillance. Le reviseur est chargé d'exercer une surveillance active sur les sociétés qui l'ont désigné. En cas d'infractions et dans certains cas particuliers, il en informe le conseil d'administration et la Commission dans un rapport. Dans les deux systèmes, les banques choisissent librement leur reviseur parmi la liste de professionnels assermentés. Cette forme de sous-traitance du contrôle à des agents privés est, dans les deux pays, parfois remise en question en raison du manque d'indépendance entre contrôleur et contrôlé(66).

Ces nombreuses analogies et ressemblances entre les deux règlementations bancaires ont d'ailleurs été parfois relevées, quoique discrètement, par les observateurs contemporains. Aussi Franz De Voghel (1903-1995), l'un des premiers spécialistes du droit bancaire belge et premier secrétaire de la Commission bancaire, écrit-il en 1938: "La législation bancaire suisse s'apparente étroitement à la législation bancaire belge. Les auteurs de cette dernière, en 1935, ont manifestement été inspirés par les réglementations adoptées en Suisse en 1934 ». En conclusion de son propos, il ajoute même: «La législation bancaire suisse est, avec la législation bancaire belge, une des plus libérales qui soient. On ne saurait envisager un contrôle légal des banques avec une immixtion moindre de l'État. Ces deux législations sont sœurs. ॥ ${ }^{(67)}$. Quelques autres experts de l'époque ont relevé ce lien de parenté(68).

(63) Les uniques liens légaux entre les deux commissions et l'État ou la banque centrale ont trait à leur financement, à leur composition et la publication annuelle d'un rapport d'activité.

(64) A.M. AlLen, Commercial Banking Legislation, op. cit., p. 40-47.

(65) Cf. J. ZAHN, Die Bankaufsichtsgesetze, op. cit., p. XXVI.

(66) Cf. sur la Suisse: Th. GIDDEY, "Gendarme ou médecin des banques?», op. cit., p. 153-157; sur la Belgique: Jean LE BRUN, «Nature et modalités juridiques du contrôle revisoral des banques», dans Reflets et Perspectives de la Vie économique, $\mathrm{n}^{\circ} 1,1971$, p. 31-51, ici p. $48-51$.

(67) Fr. De Voghel, «La législation bancaire suisse», op. cit., p. 4 et 27.

(68) L'observateur italien Felice Setti relève par exemple que «Le contrôle des banques de dépôts introduit en Belgique par arrêté royal du 9 juillet 1935 présente quelque analogie avec celui de la Suisse». Felice SETTI, «La protection de l'épargne et la réglementation du crédit dans la législation étrangère», dans Rivista Bancaria, 1937, p. 1-69, ici p. 41. Michel 
Dans la presse également, certains commentateurs confirment les nombreuses analogies. En Suisse, le Journal de Genève, quotidien libéral proche des milieux financiers genevois, se borne à relever que certaines dispositions «se rapprochent», «rappellent», voire «sont empruntées» à la récente loi fédérale suisse ${ }^{(69)}$. Dans la presse financière belge, les références à la loi bancaire helvétique l'élève au statut de modèle à suivre. Dans une série d'articles publiés dans la revue financière Le pour et le contre, l'on vante ouvertement les mérites de la réglementation suisse: "C'est un modèle d'ordre qui s'inspire d'une saine technique: tout ce qui est de marque helvétique est généralement bon et bien fait». Plus loin, l'auteur en conclut que «la loi helvétique est parmi toutes les autres lois similaires de l'étranger, celle qui doit le plus retenir l'attention; d'abord par ses qualités propres qui sont hors pair et aussi à cause des circonstances qui l'on fait naître» (70). Même son de cloche, dans un autre contexte, en janvier 1939, lorsque la Gazette, quotidien libéral bruxellois, exige un assouplissement du contrôle bancaire belge. Alors même qu'il est conscient du rôle de modèle qu'a joué la loi fédérale en 1935, l'auteur regrette les directions différentes qu'ont prises les deux régimes:

"Quel abîme entre la manière dont les législateurs du pays des vingt-deux cantons ont compris le problème et celle que nos impénitents dirigistes ont fait prévaloir! Nous disons bien d'un côté, les 'législateurs', et de l'autre, les 'dresseurs de plans', ce qui constitue de prime abord une dissemblance essentielle, car les deux systèmes diffèrent du tout au tout, du chef que l'un a été conçu en vue d'une collaboration avec les banques et l'autre en vue d'une action contre elles. L'esprit de la loi suisse est bienveillant et constructif; quant à celui de la loi belge, nous l'avons suffisamment critiqué dans nos articles précédents pour ne plus nous y appesantir; disons cependant qu'il est tracassier, vindicatif, empreint d'animosité et déplorablement destructif» ${ }^{(71)}$.

Cette prise de position caricaturale et manichéiste est en contradiction avec les fortes ressemblances, en comparaison internationale, entre les deux systèmes. Elle vise bien plus à justifier une dérégulation du secteur bancaire belge et une remise en question du rôle de la Commission bancaire. Il reste significatif que le modèle helvétique est à nouveau cité en exemple par un ardent critique de l'interventionnisme étatique.

Charvet souligne aussi que «la Commission bancaire n'est pas, comme on le voit, sans présenter d'étroites analogies avec la Commission fédérale des Banques, créée par la loi bancaire suisse du 8 novembre 1934, qui a fréquemment inspiré le législateur belge». M. Charvet, La crise du système, op. cit., p. 132, n. 1.

(69) T.B., «Le contrôle des banques en Belgique», dans Journal de Genève, 11, 14 et 19 juillet 1935, p. 7.

(70) U.P., «Réforme des bilans de banques - Facteur essentiel du contrôle des banques en Belgique», dans Le Pour et le Contre, journal financier belge, 14 et 28 avril 1935.

(71) Non signé, «Nouvelle contribution à la réforme de la loi bancaire. C'est de Suisse à présent que nous vient la lumière», dans La Gazette, 8 janvier 1939. 


\section{Un modèle pour qui, pour quoi?}

Une fois établi le lien de parenté - ou de filiation - entre les législations bancaires belge et suisse, il reste à éclaircir la question des motivations. En quoi la loi fédérale sur les banques de novembre 1934 et le système de contrôle bancaire qui en est issu ont-ils été un modèle pour les banquiers et, dans un second temps, les législateurs belges? Car, rappelons-le, les sources archivistiques indiquent bel et bien que, chronologiquement, c'est le comité de banquiers présidé par Alexandre Galopin qui a, le premier, plaidé en faveur d'un système inspiré de la Suisse ${ }^{(72)}$. Il convient d'emblée de rejeter la réponse qui est mise en avant dans de nombreux cas de transferts de politiques publiques. La régulation bancaire helvétique n'a pas constitué un modèle à imiter par ses qualités intrinsèques et les résultats efficaces qu'elle a montrés. Entrée en vigueur le $1^{\mathrm{er}}$ mars 1935, la loi bancaire suisse est si récente au moment de l'élaboration du contrôle bancaire belge qu'il serait difficile de prétendre que les législateurs belges ont cherché à s'inspirer d'un régime de surveillance aux rouages bien huilés, ayant fait les preuves de ses mérites. En effet, au moment où une «Commission bancaire» fait son apparition dans les projets belges, le 16 juin 1935, la Commission fédérale des banques dont elle est inspirée vient de vivre la sixième séance de sa courte existence $^{(73)}$. Davantage que les résultats produits par le système helvétique, les législateurs et banquiers belges se sont sans doute inspirés des principes fondamentaux qui caractérisent la surveillance bancaire helvétique mise en place en 1934.

Un premier élément de réponse réside dans les conditions similaires qui ont amené à l'aboutissement d'une loi bancaire. En Suisse comme en Belgique, les impulsions législatives trouvent leur origine dans une grave crise bancaire ${ }^{(74)}$. Sur les huit grandes banques que comptent la Suisse en 1930, seules deux, le Crédit suisse et la Société de Banque suisse, traversent les années 1930 sans devoir mener des opérations d'assainissement ou de restructuration. Entre 1930 et 1938, environ un sixième des institutions bancaires du pays (soixante établissements) font faillite ou sont contraintes à la fusion ${ }^{(75)}$. En décembre 1933, lorsque le gouvernement fédéral demande au Parlement l'approbation d'un plan de sauvetage de la Banque populaire suisse impliquant une prise de participation de la Confédération équivalant à près du quart de ses dépenses cette année-là, la pression politique en faveur

(72) Cf. AGR, Papiers Max-Léo Gérard, dossier 31, lettre de Max-Léo Gérard à Paul Van Zeeland, 4 juin 1935.

(73) La sixième séance de la Commission fédérale des banques a lieu le 15 juin 1935, à peine dix semaines après la première séance de son existence le 8 avril. Cf. AF, E 6250 (A), 1000/1059, vol. 6 : procès-verbaux des séances 1935-1936.

(74) Sur la crise bancaire en Suisse: Paul EHRSAM, «Die Bankenkrise der 30er Jahre in der Schweiz», dans Urs Zulauf, ed., 50 ans de surveillance fédérale des banques, Zurich, Schulthess, 1985, p. 83-118; Jan BAUMANN, Bundesinterventionen in der Bankenkrise 1931-1937. Eine vergleichende Studie am Beispiel der Schweizerischen Volksbank und der Schweizerischen Diskontbank, Université de Zurich, 2007.

(75) Malik MazBouri, Sébastien Guex \& Rodrigo Lopez, «Finanzplatz Schweiz», dans Patrick Halbeisen, Margrit Müller \& Béatrice VeYrassat, eds., Wirtschaftsgeschichte der Schweiz im 20. Jahrhundert, Bâle, Schwabe, 2012, p. 467-518, ici p. 490. 
d'une réglementation du secteur se renforce considérablement. Comme en Belgique quelques mois plus tard, l'adoption d'un régime de supervision bancaire s'apparente à une forme de compromis politique. D'une part, les socialistes, dans un premier temps partisans d'un contrôle bancaire donnant aux représentants de l'État une influence sur la direction du crédit, revoient leurs exigences à la baisse. D'autre part, les milieux bancaires et les défenseurs du libéralisme économique acceptent le principe d'une législation mais souhaitent y limiter les prérogatives de l'État au minimum. Pour les auteurs de la loi helvétique, cette dernière est d'ailleurs « dépourvue de tout caractère étatiste, puisque le contrôle est confié à l'initiative privée, que la commission des banques instituée par la loi n'est pas une section de l'administration et que cet organe n'a pour ainsi dire pas de droit de regard dans le ménage des banques ni dans les rapports de revision» ${ }^{(76)}$. Il semble donc bien que le fameux compromis politique mis en avant tant dans le cas belge que suisse ${ }^{(77)}$ ait débouché sur un régime qui est en parfaite adéquation avec les vœux des milieux qui font l'objet de la régulation. Dans cette perspective, les dirigeants bancaires belges auraient vu d'un bon œil une solution législative peu contraignante et née dans des circonstances proches. Ajoutons également qu'il ne s'agit pas, pour les législateurs et banquiers belges, de se soumettre servilement à un modèle étranger. La dynamique semble plutôt indiquer que, face à la nécessité d'élaborer rapidement un projet de loi réduisant l'intervention étatique au minimum, de Barsy et le comité de banquiers, adoptant une approche pragmatique, ont trouvé dans la loi suisse sur les banques une source d'inspiration opportune, apte à résoudre leurs problèmes.

En outre, il faut souligner que le choix d'un système de surveillance supervisé par un organe de contrôle indépendant et des reviseurs privés, s'il trouve son inspiration dans l'exemple helvétique, répond idéalement aux besoins des intérêts bancaires belges pour les deux raisons suivantes au moins.

D'une part, la création de toute pièce d'un organisme autonome permet d'éviter d'accroître les compétences de la Banque nationale de Belgique. En effet, au moins depuis l'échec du plan Janssen de stabilisation du franc en 1926, l'autorité monétaire a occupé une position plus en retrait dans les relations avec l'État belge, au profit de la Société générale, qui devient, sous l'influence d'Émile Francqui, l'interlocuteur principal du monde bancaire ${ }^{(78)}$. En 1935, au moment de l'élaboration du contrôle bancaire, la banque centrale est donc reléguée à un rôle secondaire. Dans son analyse détaillée du processus d'élaboration, Vanthemsche estime que la mise de côté de la Banque nationale de Belgique en tant qu'autorité de surveillance est le

(76) Paul Rossy \& Robert Reimann, Commentaire de la loi fédérale du 8 novembre 1934 sur les banques et les caisses d'épargne et du règlement d'exécution du Conseil fédéral du 26 février 1935, Zurich, Éd. Polygraphiques, 1935, p. 10.

(77) Pour la Belgique: M.-L. GÉRARD, «À propos d'un anniversaire», op. cit., p. 33; Franz De Voghel \& A[ntoinette] GRÉGOIRE, Le statut légal des banques et le régime des émissions. Textes légaux et réglementaires et rapports de la Commission bancaire coordonnés, Bruxelles, Bruylant, 1949, préface d'Eugène de Barsy, p. IX-X. Pour la Suisse: Th. GIDDEY, «Gendarme ou médecin des banques?», op. cit., p. 148.

(78) G. KuRgan-VAn HENTENRYK, "The Banque nationale de Belgique», op. cit., p. 121. 
résultat d'un double rejet, à priori contradictoire ${ }^{(79)}$. A la fois les banques privées, qui craignent une banque centrale trop envahissante, et les législateurs progressistes de l'OREC, qui se méfient de l'emprise indirecte des banquiers privés dans la banque centrale, veulent tenir cette dernière à l'écart du contrôle bancaire. Max-Léo Gérard semble avoir joué un rôle important dans ce court-circuitage ${ }^{(80)}$. En Suisse aussi, la solution de la Commission fédérale des banques est présentée comme une solution par défaut, «un mal nécessaire», suite au refus volontaire de la Banque nationale suisse, pour diverses raisons, d'endosser le rôle de gendarme des banques ${ }^{(81)}$. En ce sens, il faut aussi rappeler que, dans la perspective à plus long terme des rapports entre État et banques, la phase réglementaire des années 1930 ne représente pas une rupture. Au contraire, en Belgique comme en Suisse, les processus d'élaboration d'une réglementation bancaire mettent en exergue la suprématie des intérêts des banques privées par rapport à ceux de la banque centrale et de l'État.

D'autre part, l'instauration d'une commission indépendante, formée de techniciens émanant en partie des milieux contrôlés, et non d'un office central employant des fonctionnaires, répond au besoin des banques de ne pas effrayer sa clientèle sur la portée du contrôle et le caractère confidentiel des opérations bancaires. Cette préoccupation est clairement exprimée dans une note commune des banques belges datant du 19 juin 1935 :

«L'État, sans parler des banques elles-mêmes, subirait, du point de vue de l'économie nationale, le plus grand dommage si les clients des banques, à l'occasion de l'instauration d'un contrôle, perdaient la confiance qu'ils ont conservée jusqu'ici, dans le caractère strictement confidentiel des opérations qu'ils confient à leurs banquiers. C'est sous l'angle de cette considération qu'il faut résoudre le problème de l'organisation du contrôle de l'application de l'arrêté royal et des règlements établis en suite de celui-ci et des sanctions qu'il comporterait. [...] On peut poser en fait - et cependant cette solution doit être écartée - qu'il serait extrêmement difficile de constituer pareille organisation en choisissant les éléments qui la composent en-dehors de ceux-là même qu'il s'agit de contrôler. Que si d'autre part l'organisation de contrôle était composée de fonctionnaires placés sous la dépendance de l'Etat et quel que soit d'ailleurs le caractère irréprochable de ces fonctionnaires, les clients tant belges qu'étrangers hésiteraient à croire que la nature confidentielle de leurs relations avec leurs banquiers serait encore sauvegardée ${ }^{(82)}$.

Ainsi, dans l'esprit des dirigeants de banque belges, l'établissement d'une autorité de surveillance indépendante de l'État vise également à préserver la confiance des déposants, et à éloigner le spectre d'une institution purement étatique au regard inquisiteur. Là aussi, le parallèle avec la mise en place

(79) G. VANTHEMSChe, «L'élaboration de l'arrêté», op. cit., p. 428. Cf. aussi H. VAN DER WEE \& K. TAVERNIER, La Banque nationale de Belgique, op. cit., p. 298.

(80) G. Kurgan-van Hentenryk, Max-Léo Gérard, op. cit., p. 160-162; G. VANTHEMSCHE, «L'élaboration de l'arrêté», op. cit., p. 427.

(81) Th. GIDDEY, «Gendarme ou médecin des banques?», op. cit., p. 149-151.

(82) ABNB, O303/1, «Note sur le 'Projet de contrôle des banques'», 19 juin 1935, p. $5-6$. 
en Suisse de la Commission fédérale des banques est frappant. En 1931, le Conseiller fédéral en charge des Finances conservateur, Jean-Marie Musy (1876-1952), ne tenait-il pas un discours étonnamment proche de celui des banquiers belges évoqué ci-dessus:

«La banque peut-elle, doit-elle être placée sous le contrôle officiel de l'État? Cela ne paraît pas nécessaire. [...] L'intervention des contrôleurs officiels inquiéterait peut-être, à tort ou à raison, la clientèle qui attache une très grande importance à la discrétion sur laquelle elle veut pouvoir compter. La fuite des capitaux déposés dans nos banques qui pourrait être la conséquence de l'institution d'un contrôle officiel causerait à notre économie nationale un mal dont le peuple tout entier aurait à souffrir» ${ }^{(83)}$.

Partageant les inquiétudes des autorités suisses à l'égard des inspections étatiques et de leurs potentielles nuisances sur la capacité d'attraction du capital, les banquiers belges ont cherché à promouvoir un système proche de celui instauré en Suisse. Sur ce point précis, la réglementation belge n'offre cependant pas de protection comparable à celle du secret bancaire helvétique. Malgré une confidentialité renforcée à l'égard des membres de la Commission bancaire et des reviseurs, la réglementation belge n'intègre pas de dispositions particulières sur le secret professionnel des banques.

Un dernier élément à mettre en avant parmi les raisons qui ont pu inciter les législateurs belges à tourner leur regard vers la Suisse réside dans l'influence des idées corporatistes. Plus haut, nous avons évoqué que la composition de la Commission bancaire, de l'aveu même du Premier ministre Van Zeeland, avait été inspirée de la loi suisse. Les auteurs de la loi belge ont repris le principe d'une participation de spécialistes de la technique bancaire ou revisorale et l'ont développé en précisant que deux des six membres doivent être désignés sur une liste fournie par les représentants des banques. En ce sens, les législateurs belges ont inséré une touche de corporatisme ${ }^{(84)}$, c'est-à-dire une forme d'organisation socio-économique reposant sur les corporations professionnelles. Cette idéologie, souvent présentée comme une «troisième voie» entre le socialisme et le capitalisme libéral, connaît un essor important dans les années 1930. Durant l'élaboration de la loi bancaire suisse, certains parlementaires catholiques-conservateurs avaient réclamé la mise sur pied d'une organisation interne à la profession bancaire chargée d'imposer une assurance des dépôts et d'exclure les banques récalcitrantes de la corporation ${ }^{(85)}$. Mais cette forme d'autorégulation ne rencontra pas de succès et les projets n'aboutiront pas. En revanche, tout au long du processus de décision, l'influence de l'Association suisse des Banquiers, fondée en

(83) Jean-Marie MuSY, «La question du contrôle des banques», procès-verbal de la $18^{\mathrm{e}}$ Assemblée générale de l'Association suisse des Banquiers, 12 septembre 1931, p. 34.

(84) Franz De Voghel, secrétaire de la Commission bancaire, estime que l'on voit «poindre la représentation des intérêts professionnels» dans la loi belge. À ce titre, celleci se situe entre la réglementation suisse (moins corporatiste) et les lois roumaines et espagnoles, qui adoptent plus franchement le principe de l'autorité professionnelle. Fr. DE VOGHEL, Contrôle des banques, op. cit., p. 131-133.

(85) H. BÄNZIGER, Die Entwicklung der Bankenaufsicht, op. cit., p. 120-121; Thibaud GIDDEY, La genèse et les premières années d'activités de la Commission fédérale des Banques (1931-1943), mémoire de Maîtrise, Université de Lausanne, 2010, p. 57. 
1912, est permanente ${ }^{(86)}$. En Belgique aussi, les idées corporatistes ont le vent en poupe. Comme le relève Moreau, plusieurs voix s'élèvent en 1935 pour regretter l'absence d'une organisation professionnelle des banquiers qui aurait pu promouvoir l'autodiscipline au sein du secteur et éviter l'intervention de l'Etat ${ }^{(87)}$. Il est d'ailleurs remarquable - et c'est là un fait qui n'a été que trop rarement mentionné par les historiens - qu'en Belgique le processus d'élaboration d'une régulation bancaire n'ait pas été influencé par une industrie bancaire organisée au sein d'une association d'intérêts, mais plutôt par une étroite collaboration avec un cercle réduit de représentants de certains grands établissements. Le mouvement est même inverse. C'est sous l'impulsion de la promulgation d'un contrôle bancaire en juillet 1935 qui prévoyait une "réunion des représentants des banques » ${ }^{(8)}$, que l'Association belge des Banques voit le jour en décembre 1936. Plus précisément, c'est la conjonction de deux facteurs qui est à l'origine de la naissance de ce groupement professionnel ${ }^{(89)}$. D'une part, la nécessité pour les milieux bancaires de présenter un front uni face aux autorités étatiques et parastatales et de former un «partenaire de concertation» fédéré. D'autre part, dans le sillage des importants mouvements sociaux et grèves de juin 1936, les banques sont invitées à être représentées dans les nouvelles commissions paritaires mises en place pour résoudre les conflits sociaux au sein des entreprises. Dans ce contexte, l'ABB est conçue à la fois comme une trade association, chargée de l'organisation des marchés avec les interlocuteurs extérieurs et des conventions internes au secteur, et comme employers' association, responsable des rapports de travail ${ }^{(90)}$. Sans trop entrer dans les détails, il faut ici relever que, d'après la correspondance conservée aux AGR, l'initiative première menant à la constitution de l'Association belge des Banques est l'œuvre conjointe de Georges Janssen (1892-1941), le premier président de la Commission bancaire, et Alexandre Galopin, gouverneur de la Société générale de Belgique $^{(91)}$. Parmi les divers avant-projets des statuts de l'association, l'on trouve notamment un projet non daté d'un certain Helbronner, associé de la banque privée Philippson, qui reconnaît avoir étudié, sans pour autant l'avoir

(86) Ce groupe d'intérêts est consulté et représenté dans toutes les commissions de discussion, pré-parlementaires, comme parlementaires, entre mars 1933 et mars 1935. Cf. Th. GIDDEY, La genèse, op. cit., p. 36-51.

(87) J.-L. MOREAU, «Contrôler sans nationaliser», op. cit., p. 110.

(88) «Arrêté royal $\mathrm{n}^{\circ} 185$ du 9 juillet 1935 sur le contrôle des banques et le régime des émissions de titres et valeurs», dans Moniteur belge, 10 juillet 1935, art. 37.

(89) Sur les origines de l'ABB: Michel De SMET, ed., Association belge des Banques 1936-1986, Bruxelles, Association belge des Banques, 1986, p. 7-8, 43, 51-53; Étienne ARCQ, L'Association belge des Banques, Bruxelles, Centre de Recherche et d'Information socio-politiques, 1989, p. 12-14; Commission bancaire 1935-1960, op. cit., p. 48-49; Adrien P. Timmermans, Les banques en Belgique (1946-1968), Courtrai, Groeninghe, 1969, p. 563.

(90) Sur cette distinction: Luca LANZALACO, «Business Interest Association», dans Geoffrey Jones \& Jonathan ZEITLIN, eds., Oxford Handbook of Business History, Oxford, Oxford University Press, 2008, p. 293-315.

(91) AGR, Commission bancaire, financière et des assurances (510-2119), $\mathrm{n}^{\circ} 220$ : Dossier de principe 1935-1939, Lettres échangées en Georges Janssen et Alexandre Galopin, 8 et 11 juillet 1936. 
«copié servilement»l'organisation professionnelle des banques suisses ${ }^{(92)}$. Son projet d' «Union professionnelle des Banquiers belges», reprend ainsi le modèle helvétique ${ }^{(93)}$ en préconisant une association individuelle des banquiers et non une affiliation des sociétés. Bien que les statuts finaux de l'ABB du 2 décembre 1936 ne s'en inspirent que peu, ce nouvel exemple confirme la prégnance du modèle d'organisation helvétique parmi les élites bancaires belges.

\section{Commission bancaire et Commission fédérale des banques: la carotte ou le bâton?}

À partir de ces parentés de naissance, comment les régimes de surveillance bancaire belge et suisse vont-ils évoluer durant leurs premières années d'existence? En dépit de l'intérêt majeur des études de droit comparé qui se limitent à apprécier les normes telles qu'elles figurent dans les textes de loi, il est souvent encore plus révélateur d'examiner l'application effective de ces mêmes normes. Lors de l'entrée en vigueur des nouvelles réglementations, dans les deux cas, les mesures d'adaptation de la part du secteur bancaire ne sont pas radicales. En Suisse, les promoteurs et commentateurs de la loi s'accordent à dire que celle-ci, dans l'ensemble, ne fait que généraliser à l'ensemble du secteur des pratiques déjà existantes chez la plupart des banques ${ }^{(94)}$. En Belgique, même chez les plus grandes banques, les changements sont de faible portée. Un aide-mémoire énumérant les dispositions à prendre avec l'entrée en vigueur de la loi, conservé dans les archives de la Banque de la Société générale, est significatif à ce titre ${ }^{(95)}$. Mis à part quelques retouches du bilan (constitution d'un fonds de réserve légal) en

(92) AGR, Commission bancaire, financière et des assurances (510-2119), $\mathrm{n}^{\circ} 220$ : Dossier de principe 1935-1939, Projet Helbronner (Philippson), Union professionnelle des Banquiers belges, non daté.

(93) Sur l'histoire de l'Association suisse des Banquiers: Sébastien GuEX \& Malik MAZBouRI, «De l'Association des représentants de la banque en Suisse (1912) à l'Association suisse des Banquiers (1919). Genèse et fonctions de l'organisation faîtière du secteur bancaire suisse», dans Danièle Fraboulet \& Pierre Vernus, eds., Genèse des organisations patronales en Europe (19e-20e siècles), Rennes, Presses universitaires de Rennes, 2012, p. 205-225; Sébastien GUEX \& Malik MAZBOURI, «Une grande association patronale dans la sphère publique: l'exemple de l'Association suisse des Banquiers (de 1912 à nos jours)», dans Danièle FraboulET, Clotilde DrUElle-Korn \& Pierre VernUS, eds., Les organisations patronales et la sphère publique, Rennes, Presses universitaires de Rennes, 2013, p. 205-235.

(94) Cf. «Message du Conseil fédéral à l'Assemblée fédérale concernant le projet de loi sur les banques et les caisses d'épargne, (du 2 février 1934)», dans Feuille fédérale, 1, 1934, p. 173: «Le but de la loi que nous proposons est d'étendre à toutes les banques l'obligation et le bénéfice du contrôle indépendant que se sont volontairement imposé la plupart de nos établissements financiers». Cf. aussi Max CLUSEAU, La réglementation des banques. Économie libérale ou économie dirigée? Étude critique de quelques expériences étrangères récentes, Paris, Sirey, 1938, p. 59: «La loi s'est contentée de sanctionner et de donner force légale à l'organisation déjà existante».

(95) ASG, Fonds Banque de la Société générale, $\mathrm{n}^{\circ}$ 982, notes de l'administrateurdélégué Willy de Munck, «Dispositions à prendre», 3 septembre 1935. 
vue de transmettre mensuellement une situation bancaire à la Commission bancaire, il s'agit de faire inscrire la société à la liste des banques, d'examiner les cas d'incompatibilités chez les dirigeants et de nommer le ou les reviseurs assermentés qui contrôleront la banque. Devant son conseil d'administration, le président de la BSGB, Alexandre Galopin, ne déclare-t-il pas une semaine après l'adoption de l'arrêté : "Les suggestions des banques ont été adoptées sur de nombreux points et la nouvelle réglementation, si elle est appliquée avec la compétence et le discernement requis, ne paraît pas devoir apporter à l'activité des banques des restrictions inopportunes et susceptibles de nuire à l'intérêt général» ${ }^{(96)}$.

Pour développer cette étude comparative des surveillances bancaires belges et suisses, il est judicieux de se pencher sur le rôle joué par la Commission bancaire et la Commission fédérale des banques. En effet, ce sont ces organes qui constituent le résultat le plus tangible de l'influence helvétique sur la législation belge, la Commission bancaire ayant été façonnée sur son homologue suisse. Au cours de leurs premières années d'activité, ces deux autorités de surveillance connaîtront à la fois des évolutions divergentes et des caractéristiques analogues sous de nombreux aspects. Bornons-nous ici à en mentionner superficiellement quelques-unes.

Premièrement, la composition des deux organes se fait selon des modalités semblables. Malgré, dans les deux cas, une forme de corporatisme voulue par un choix de spécialistes indépendants, la Commission fédérale des banques et la Commission bancaire se distinguent dans le profil des premiers membres nommés. Parmi les cinq premiers commissaires suisses, on trouve deux anciens banquiers issus des plus grandes banques, un parlementaire spécialisé dans les réorganisations bancaires, ainsi qu'un banquier ayant fait carrière à la banque centrale avant de collaborer à l'élaboration de la loi bancaire ${ }^{(97)}$. Le premier président de la Commission fédérale des banques n'est autre que l'ancien Conseiller fédéral - la plus haute fonction exécutive du système politique suisse - Edmund Schulthess. Malgré la présence à Berne d'un ancien ministre et d'un sénateur encore en fonction, la composition de la Commission bancaire belge semble avoir été davantage influencée par des critères politiques. Georges Janssen, un professeur de droit et ancien dirigeant de la Mutuelle Solvay puis de la Société belge de Banque, en est nommé président. Parmi les six autres membres, un grand patron industriel, un juge membre de la Chambre de Commerce international, un administrateur-délégué de la Banque du Congo belge, un directeur de la Banque nationale, et deux dirigeants de sociétés coopératives socialistes ${ }^{(98)}$. On peut émettre l'hypothèse que l'équilibre politique de cette composition reflète en

(96) Alexandre Galopin, procès-verbaux du conseil d'administration de la Banque de la Société générale, 16 juillet 1935, cité dans Guy VANTHEMSCHE, "La Banque de 1934 à nos jours», dans Herman VAN DER WEE, ed., La Générale de Banque. 1822-1997, Bruxelles, Racine, 1997, p. 287-526, ici p. 306.

(97) Edmund Schulthess (1868-1944), président, Paul Rossy (1896-1973), viceprésident, Carl Brüderlin (1873-1945), Emil Walch (1873-1965) et Albert Zust (1874-1952).

(98) Georges Janssen (1892-1941), Léon Antoine Bekaert (1891-1961), Alfred de Brouckère (1873-1948), René Guillaume (1884-1959), Louis-Jean Mahieu (1899-1940), Julien Papart (1889-1965) et André Huyssens (1888-1978). 
partie les rapports de force politiques au sein du cabinet Van Zeeland à l'été $1935^{(99)}$. Une autre différence a trait à l'écart générationnel. Les commissaires belges sont relativement jeunes, le passage à la Commission bancaire étant parfois un tremplin vers une carrière dans l'élite financière et administrative du pays. En Suisse, l'on confie la surveillance bancaire à des techniciens plus mûrs, fraîchement retraités de postes dirigeants dans le monde bancaire. Dans les deux cas, relevons que les critères linguistiques et d'origine régionale, sans être explicitement exprimés, entrent en compte dans la nomination des membres.

Deuxièmement, les deux institutions connaissent des développements comparables dans leurs relations avec l'autorité monétaire. En Suisse, les relations entre la Commission fédérale des banques et la Banque nationale suisse sont dans un premier temps particulièrement tendues, en particulier suite aux refus de la banque centrale de transmettre à la Commission les informations qu'elle obtient des banques privées ${ }^{(100)}$. En Belgique également, la collaboration n'est pas immédiate ${ }^{(101)}$. Les conceptions divergentes sur la politique monétaire entre Georges Janssen et le gouverneur Louis Franck rendent la coopération entre les deux institutions difficile. D'abord logées dans les locaux de la Banque nationale de Belgique et financée entièrement par elle, la Commission bancaire s'en affranchit rapidement. En février 1937, les différences de vues s'accentuent lorsque Janssen élabore un projet visant à étendre la surveillance de la Commission bancaire aux établissements publics et parastataux, dont la Banque nationale ou encore l'Institut de Réescompte et de Garantie. Louis-Jean Mahieu, qui a une position ambiguë puisqu'il est à la fois directeur de la banque centrale et membre de la Commission bancaire, s'y oppose nettement. Cela reviendrait paradoxalement, selon ses dires, à soumettre la Banque nationale «au contrôle qu'elle aurait dû assumer ellemême »(102). Le projet n'aboutit pas. Dès 1938, lorsque Janssen est nommé gouverneur de la Banque nationale et doit quitter, suite à une campagne de presse à son encontre alimentée par Max-Léo Gérard ${ }^{(103)}$, ses fonctions à la Commission bancaire, les deux autorités se rapprochent.

Troisièmement, la Commission fédérale des banques et la Commission bancaire opèrent durant leurs premières années avec des moyens similaires. Tandis que l'organisme suisse occupe en 1935 sept employés salariés, l'institution belge en compte neuf (en incluant le président et les secrétaires

(99) Selon Hubert Ansiaux, la candidature de Georges Janssen à la présidence de la Commission aurait été proposée et soutenue par Paul-Henri Spaak (1899-1972), son «ami et collaborateur». Cf. Hubert AnSIAUX, "Georges Janssen», dans Nouvelle Biographie nationale, t. 1, Bruxelles, Académie royale des Sciences, des Lettres et des Beaux-arts de Belgique, 1988, p. 192-199.

(100) Th. GIDDEY, «Gendarme ou médecin des banques?», op. cit., p. 150-153.

(101) H. VAN DER WeE \& K. TAVERnier, La Banque nationale de Belgique, op. cit., p. 298-299.

(102) ABNB, J 327/3, lettre de Louis-Jean Mahieu à Georges Janssen, 12 février 1937.

(103) Cf. AGR2, SGB4, fonds "secrétariat général», n 696, sous-dossier «Janssen et cumul des mandats ». Sur le rôle de Max-Léo Gérard dans le départ de Georges Janssen de la Commission bancaire: G. Kurgan-VAn Hentenryk, Max-Léo Gérard, op. cit., p. $179-180$. 
dactylographes $)^{(104)}$. Corollaire logique de cette faiblesse en personnel, les budgets des deux institutions sont relativement restreints. Malgré toutes les précautions à prendre dans la comparaison de tels chiffres, il semble que les moyens financiers de la Commission bancaire soient en 1936 un peu plus importants que ceux de la Commission fédérale des banques ${ }^{(105)}$. Les modes de financement varient également d'un pays à l'autre ${ }^{(106)}$. Malgré ces divergences, il faut retenir que les deux organes disposent, dans les deux cas, de moyens très faibles.

Ces carences sont en grande partie dues au système même de surveillance, qui confie à des reviseurs privés la tâche essentielle du contrôle comptable régulier de la société. Dans ce domaine également, la Commission bancaire et la Commission fédérale des banques connaissent certaines difficultés. En effet, ce sont elles qui sont chargées de reconnaître quels reviseurs ou sociétés de revision sont autorisés à pratiquer le contrôle bancaire. En Suisse, la question de l'indépendance des sociétés de revision vis-à-vis des banques qu'elles sont censées contrôler fait débat. Il s'avère que chez les grandes banques, la plupart des sociétés fiduciaires chargées de la revision ont été fondées par ces mêmes banques et en sont encore très proches ${ }^{(107)}$. En Belgique, c'est davantage la question de la compétence des reviseurs qui est discutée. Dans un premier temps, la Commission bancaire avait en effet décidé d'octroyer le statut de reviseur assermenté à un nombre élevé de candidats. En 1939, un changement se dessine dans la politique d'agréation de la Commission. Les récents scandales bancaires de la Caisse générale de Reports et de Dépôts et du Crédits anversois ont montré les limites du système de surveillance. Dans son rapport annuel pour 1939, la Commission bancaire avoue que «le régime des reviseurs [...] n'assurait pas au contrôle des banques l'efficacité désirable». Plus précisément, le second président de la Commission bancaire Maurice Frère (1890-1970) confirme dans une lettre au procureur du Roi à la fois l'incompétence et le manque d'indépendance du reviseur en charge de la Caisse générale de Reports et de Dépôts, une filiale de la Société Générale:

«Le reviseur a toléré sans l'en aviser, l'existence d'un risque tout à fait hors de proportion avec les dépôts confiés à la banque, risque que les docu-

(104) Pour la Suisse: Archives fédérales, E 6520 (A), 1983/50, vol. 13, Rapport d'activité annuel de la Commission fédérale des Banques, 1935. Pour la Belgique: ABNB, B 602/3, Comptabilité de la Commission bancaire, avril 1936.

(105) 1.130.283 francs belges pour la Commission bancaire contre 83.287 francs suisses (soit environ 727.000 francs belges selon le taux de change à vue, moyenne annuelle 1936, publié par la BNS: Banque nationale suisse, Manuel statistique du marché financier suisse, Zurich, Schulthess 1944, p. 46-49). Sources: pour la CB: rapport annuel 1937, pour la CFB: AF, EI (-), -/--, vol. 78 (Archives de la chancellerie fédérale), dossier 398, Eidgenössische Sparkommission, 31 janvier 1938.

(106) En Suisse, malgré des propositions visant à faire participer les banques, la Commission fédérale des Banques est entièrement financée par l'État fédéral. En Belgique, la Commission bancaire, en plus de la participation annuelle fixe de la Banque nationale, perçoit des droits sur les émissions de titre et de valeurs qu'elle examine. Dès 1937, une troisième source de revenus est prévue puisque les banques contrôlées doivent contribuer au financement proportionnellement aux montants de leurs dépôts. Cf. Arrêtés royaux n 262 du 26 mars 1936 et du 22 décembre 1936

(107) Th. GIDDEY, «Gendarme ou médecin des banques?», op. cit., p. 153-157. 
ments régulièrement communiqués à la Banque nationale ne permettaient pas d'apercevoir. En outre, elle [la Commission bancaire] a constaté certaines inexactitudes matérielles dans les documents ainsi communiqués, alors que dans ses rapports trimestriels le reviseur certifiait les avoir vérifiés. Elle a estimé enfin que d'une manière générale, le reviseur avait fait preuve dans l'accomplissement de sa mission d'un manque d'indépendance manifeste à l'égard des dirigeants de la banque ${ }^{(108)}$.

À partir de 1940, la Commission bancaire réduira progressivement le nombre des reviseurs agréés, espérant ainsi garantir une meilleure efficacité du contrôle ${ }^{(109)}$.

Quatrième parallélisme entre les deux institutions, elles semblent toutes deux opérer selon une approche semblable. Les faibles compétences légales dont elles disposent pour assurer l'application de la loi ont favorisé l'adoption, à Berne comme à Bruxelles, de méthodes plus proches de la politique de concertation, que de la «répression financière»(110). Elles pratiquent bien plus souvent la prise de contact officieuse avec les dirigeants bancaires que la prise de sanctions pénales ou la mise au pilori sous la forme d'une dénonciation publique. Ainsi, la Commission bancaire déclare-t-elle ouvertement que «la loi n'a conféré à la Commission aucun pouvoir d'intervention quant au fond, lors même que les erreurs de gestion feraient peser une menace grave sur la liquidité et la solvabilité de la banque»(111). Face à cette absence de moyens d'actions, Eugène de Barsy, auteur de la loi et président de la Commission bancaire pendant près de trente ans (1944-1972), estime que la priorité de la Commission doit être de "présenter des observations» et d' «émettre des recommandations» ${ }^{(112)}$. Il insiste sur «la collaboration confiante qui s'est établie très généralement et qui n'a cessé de s'amplifier entre la Commission bancaire et les milieux sur lesquels son action s'exerce »(113). André Bruyneel, spécialiste du droit bancaire belge et ancien collaborateur de la Commission bancaire présente son activité de la manière suivante:

(108) ABNB, J 327/17, lettre de Maurice Frère à W. Ganshof van der Meersch, 17 novembre 1939.

(109) Cf. Règlement d'agréation des reviseurs du 22 novembre 1935 modifié le 20 octobre 1939. Le nombre de reviseurs assermentés passe de 200 en 1938 à 57 en 1939, puis 28 en 1944. Cf. Rapports annuels de la Commission bancaire.

(110) L'expression anglaise financial repression désigne souvent la période de forte régulation bancaire des années 1950 aux années 1970, qui trouve son origine dans les réglementations de l'Entre-deux-guerres, marquée par des nombreuses restrictions réglementaires qui empêchent les intermédiaires financiers de développer pleinement leur potentiel. Cf. Stefano BAtTilossi \& Jaime Reis, eds., State and Financial System in Europe and the USA. Historical Perspectives on Regulation and Supervision in the Nineteenth and Twentieth Centuries, Burlington, Ashgate, 2010, p. 9.

(111) Rapport annuel de la Commission bancaire pour 1969-1970, p. 42.

(112) Dans une interview donnée à la RTB le 23 janvier 1973 et reproduite dans Eddy WYMEERSCH, ed., In bono et aequitate perseverans. Baron de Barsy 1906-1985, Bruxelles, Bruylant, 1985, p. 267.

(113) Préface d'Eugène de Barsy dans Denis Ponlot, Le statut légal des banques et le contrôle des émissions de titres et valeurs, Court-Saint-Étienne, Émile Oleffe, 1958, p. XII. 
«C'est en effet délibérément qu'une philosophie de consultation avec ses administrés inspire depuis plus de vingt ans toute l'action générale de la Commission, qui veut ainsi fonder le contrôle des banques sur l'adhésion des intéressés autant, sinon plus, que sur des textes d'ailleurs lacunaires. [...] La Commission, dans des circonstances normales, maintient des contacts permanents avec les dirigeants de chaque banque; les entretiens sont provoqués tantôt par ceux-ci, tantôt par la Commission elle-même lorsqu'elle juge nécessaire sur la base des informations recueillies ou des échanges de vues avec le reviseur. Une certaine action de conseil et de recommandation est ainsi officieusement exercée» ${ }^{(114)}$.

En Suisse également, le même type d'approche marque la politique de la Commission fédérale des banques à l'égard des sociétés soumises à son contrôle. La faiblesse de ses capacités d'intervention directes fait que, face à une banque récalcitrante qui refuse de se conformer à un de ses conseils, la Commission est pratiquement désarmée. Elle procède surtout par voie de recommandations et d'instructions informelles ${ }^{(115)}$. Daniel Bodmer, futur collaborateur de la Commission fédérale des banques, en fait le portrait suivant :

«La Commission fédérale des banques a aussi déployé une discrète activité privée consistant à conseiller des banques menacées ou, le cas échéant, à exercer sur certaines d'entre elles une bienfaisante pression psychologique. [...] D'une façon générale, elle a dû suppléer au défaut de compétences par l'action psychologique, qui est fonction du prestige qu'elle a su s'acquérir» ${ }^{(116)}$.

En Suisse comme en Belgique, les autorités de surveillance mises en place en 1934-1935 ont dû compenser des pouvoirs juridiques lacunaires par une forme de pression morale (moral suasion).

Dans ce cadre général très proche, il est cependant possible d'affiner l'analyse. Il en ressort que durant ses premières années d'activité, la Commission fédérale des banques a souvent adopté une politique plus prudente, notamment dans la définition exacte du champ d'application de la loi bancaire ${ }^{(117)}$. À la moindre incertitude au sujet de la base légale sur laquelle repose son action, l'organisme helvétique opte pour une attitude légaliste et timorée. En Belgique en revanche, sous la présidence de Georges Janssen, la Commission bancaire fait montre d'un esprit d'initiative qui inquiète souvent les milieux bancaires. Comme le relève Vanthemsche, les dirigeants de grands instituts financiers belges estiment que la Commission bancaire fait du zèle et outre-

(114) André BRuyneel, "La Commission bancaire belge», dans Revue Banque, ${ }^{\circ}$ 303-305, 1972, p. 13-25, 125-139, 247-257, ici p. 23 et 25. Sur cette même question, voir aussi J.-L. MOREAU, «Contrôler sans nationaliser», op. cit., p. 112-113.

(115) Cf. Alain HIRSCH, "La surveillance des banques en Suisse», dans Marcus LutTer, Helmut Kollhosser \& Winfried Trusen, eds., Recht und Wirtschaft in Geschichte und Gegenwart, Munich, C.H. Beck'sche Verlagsbuchhandlung, 1975, p. 461469, ici p. 465-466.

(116) Daniel BoDmer, L'intervention de la Confédération dans l'économie bancaire suisse, Bâle, National-Zeitung, 1948, p. 183.

(117) En particulier suite à un revers juridique au Tribunal fédéral en 1937, cf. Th. GIDDEY, La genèse, op. cit., p. 96. 
passe régulièrement ses compétences ${ }^{(118)}$. La volonté de la Commission d'appliquer strictement les mesures d'incompatibilités qui affectent le personnel dirigeant, ainsi que les tracasseries administratives que suscitent les demandes d'information sont mises en cause. Pour Alexandre Galopin, «le 'contrôle' dévie inéluctablement vers la 'direction'» ${ }^{(119)}$. Ces critiques sont d'ailleurs fréquemment reprises dans la presse conservatrice et financière. La Commission bancaire s'y voit affublée de noms d'oiseaux: un «vrai garde-chiourme de nos institutions financières», détenant des "pouvoirs dictatoriaux » ${ }^{(120)}$, «d'inspiration diabolique», «une entrave à l'expansion, à l'utilisation et à la création des capitaux» (121), «un organisme inopérant, coûteux et néfaste» ${ }^{(122)}$, instituant «un véritable régime inquisitorial» ${ }^{(123)}$, un «État dans l'État» ${ }^{(124)}$, utilisant des «méthodes qui n'ont rien à envier à celles en honneur, notamment chez nos voisins de l'Est» ${ }^{(125)}$. Du côté des dirigeants de la Société générale de Belgique, les critiques sont exprimées à la fois publiquement et à l'interne. Dans le rapport annuel sur l'année 1939, le grand établissement dénonce que «chaque année qui passe depuis la mise en vigueur de la nouvelle législation marque davantage ses effets délétères » ${ }^{(126)}$. Dans des notes internes, elle regrette également que la Commission bancaire adopte «les solutions les plus jacobines» et «une attitude intolérable» ${ }^{(127)}$. Ces frictions témoignent des velléités d'une partie des milieux financiers de demander la suppression ou le bridage de la Commission bancaire, considérée comme un produit du planisme d'Henri de Man, devenu inutile depuis l'apaisement de la crise bancaire.

Le cinquième et dernier point commun entre les autorités de surveillance suisse et belge renvoie aux activités concrètes exercées par les deux institutions. Une analyse des procès-verbaux des deux organismes révèle en effet que, durant leurs premières années d'existence ${ }^{(128)}$, les strictes tâches d'exécution du contrôle bancaire - c'est-à-dire notamment l'établissement de la liste des banques, des reviseurs agréés, et surtout l'analyse des rapports de revision en vue de déceler les situations mettant en danger les créanciers et la stabilité financière - n'occupent qu'une place secondaire. À Berne, la Commission fédérale des banques est principalement submergée de travail dans l'application des mesures exceptionnelles d'assainissements bancaires instaurées par la loi bancaire ${ }^{(129)}$. À Bruxelles, une importante part des débats

(118) G. Vanthemsche, «La Banque de $1934 »$, op. cit., p. 307. 1940.

(119) AGR2, ASGB4, Dossier Galopin, $n^{\circ}$ 165, lettre de Galopin à Mélot du 20 février

(120) La Gazette, 8 janvier 1939.

(121) La Gazette, 30 octobre 1938.

(122) La Nation Belge, 19 juillet 1939.

(123) Étoile Belge, 6 avril 1937.

(124) Écho de la Bourse, 12 janvier 1937.

(125) Écho de la Bourse, 19 mai 1937.

(126) Rapport annuel de la Société générale de Belgique sur l'année 1939, p. 15.

(127) ASG, Fonds BSGB, $n^{\circ}$ 984, entretien entre Maurice Frère (Commission bancaire) et Willy de Munck (BSGB), 18 novembre 1938.

(128) Pour la Commission fédérale des banques: 1935-1943, pour la Commission bancaire: $1935-1940$.

(129) Th. GIDDEY, La genèse, op. cit., p. 103-114. 
concerne l'examen des émissions de valeurs et de titres. Cette compétence fait certes partie intégrante des tâches de l'organisme, mais elle fait passer au second plan les prérogatives que l'on attribue classiquement à une autorité de surveillance bancaire. En reprenant des analogies employées par les acteurs mêmes dans diverses publications, nous pouvons affirmer que les gendarmes de banque en Suisse et en Belgique ont plutôt joué un rôle, dans le cas helvétique, de «médecin des banques» ${ }^{(130)}$, et dans le cas belge, d'un «agent de la circulation qui autorise alternativement les passages dans l'un et l'autre sens, sans pouvoir fixer la destination des véhicules qu'il contrôle» ${ }^{(131)}$.

\section{Conclusion}

À huit mois d'intervalle, deux législations réglementant l'activité des banques ont vu le jour en Suisse et en Belgique en novembre 1934 et en juillet 1935. Les nombreuses ressemblances entre les deux systèmes de régulation mis en place, et qui ont parfois interpellé les spécialistes du droit bancaire comparé, ne doivent rien au hasard. Il ressort en effet d'une analyse précise des sources à disposition que les législateurs belges, après une consultation d'un comité représentant les intérêts des grandes banques, ont considérablement modifié leurs projets de loi en s'inspirant du modèle suisse. Celui-ci apparaissait sans doute aux yeux des banquiers belges comme un cas exemplaire, car il permettait d'instaurer un contrôle très libéral, avec une immixtion moindre de l'État. En ce sens, il représentait surtout un moindre mal, face à des projets plus interventionnistes. Un certain nombre de choix institutionnels, dont celui d'instaurer une commission bancaire indépendante, résulte de cette inspiration helvétique. Au-delà du seul texte de loi, ce phénomène d'émulation a également provoqué, du moins durant leurs premières années d'existence, des évolutions parallèles dans le modus operandi des deux organismes de surveillance créés à l'occasion.

Dans le domaine de la science politique, une littérature relativement fournie a étudié les phénomènes de transfert de politiques publiques (policy transfer), de lesson drawing, ou encore de diffusion transnationale de certains exemples à imiter ou à éviter ${ }^{(132)}$. Comment qualifier, en termes plus généraux, le cas de l'influence helvétique sur l'élaboration de la réglementation

(130) Paul Rossy, «Monsieur Edmond Schulthess à la Commission fédérale des Banques», dans Fritz MANGOLD, ed., Festgabe für Bundesrat Dr. h. c. Edmund Schulthess zum siebzigsten Geburtstag am 2. März 1938, Zurich, Polygraphischer Verlag, 1938, p. 287-297, ici p. 296.

(131) Selon la formule utilisée par Georges Janssen, en commentant une métaphore de Max-Léo Gérard, dans une conférence donnée le 16 mars 1937 à la Société belge d'Études et d'Expansion à Liège, reproduite intégralement dans La Métropole du 17 mars 1937. Cf. aussi G. KuRGan-VAn HENTENRYK, «Max-Léo Gérard», op. cit., p. 677.

(132) Voir par exemple: David P. DOLOWITZ, \& David MARSH, "Who Learns What from Whom: a Review of the Policy Transfer Literature», dans Political Studies, t. 44, 1996, p. 343-357; Richard Rose, Lesson-drawing in Public Policy : a Guide to Learning Across Time and Space, New Jersey, Chatham House, 1993. Pour une critique de la pertinence de ces concepts, cf. Oliver JAMES \& Martin LODGE, «The Limitations of 'Policy Transfer' and 'Lesson Drawing' for Public Policy Research», dans Political Studies Review, 2003, vol. 1, p. 179-193. 
bancaire belge, à l'aide de cet outillage théorique? Il faut tout d'abord souligner que nous sommes en présence d'une approche peu contraignante de transfert transnational de politique, et que sa portée est limitée. Autrement dit, les législateurs belges se sont volontairement inspirés d'un exemple étranger; celui-ci n'a pas été activement promu par les autorités suisses. Il faudrait donc plutôt parler d'une forme d'importation de régime de régulation que d'une exportation à visée expansive. Dans le même sens, le cas qui nous intéresse constitue le degré minimal de convergence politique: il s'agit d'une situation plus proche d'une simple influence de certains principes et choix institutionnels que d'un cadre politique général transféré intégralement. Quant aux diverses motivations qui peuvent être à l'origine du phénomène de diffusion ou de transfert international, le cas du contrôle bancaire belge influencé par l'exemple suisse est aussi singulier ${ }^{(133)}$. Il ne s'agit pas de l'imitation d'un modèle considéré comme une pratique optimale (best practice), et choisi en fonction de l'efficacité avérée d'un exemple étranger reconnu en tant que tel. Un simple regard à la proximité chronologique permet de rejeter cette hypothèse. Il constitue plutôt un cas d'émulation permettant à un législateur de s'inspirer relativement rapidement et à moindre frais d'une solution étrangère préexistante. En revanche, - et c'est là un point à souligner - l'influence helvétique n'a pas joué un rôle de légitimation officielle de décisions déjà prises. En effet, il est significatif que les législateurs belges, tant dans le rapport au Roi du 9 juillet 1935 accompagnant l'arrêté royal, que dans les commentaires quasiment officiels de la réglementation publiés peu après son entrée en vigueur, ne mentionnent pas, même vaguement, une forme d'inspiration de modèles étrangers. Alors que dans les sources et chez certains observateurs bien renseignés les influences étrangères sont mentionnées, les discours publics restent curieusement très discrets sur cette question ${ }^{(134)}$. Comment comprendre ce silence? Une hypothèse mènerait à croire que, dans le compromis politique que constituait l'arrêté royal sur le contrôle bancaire, un système de surveillance ouvertement estampillé comme inspiré de la très libérale loi helvétique n'aurait pas fait l'unanimité. Enfin, notre cas d'étude se distingue des carcans classiques de transferts de politiques publiques ${ }^{(135)}$

(133) Colin Bennett définit cinq types de motifs politiques derrière le phénomène d'inspiration étrangère: "Elites who utilize the policy experiences of other states may have any of five different political motives: to put an issue on an institutional agenda; to mollify political pressure; to emulate the actions of an exemplar; to optimize the search for the best policy; and to legitimate conclusions already reached.» Colin BENNETT, «How States Utilize Foreign Evidence», dans Journal of Public Policy, t. 11, 1991 n 1, p. 33.

(134) En ce sens, ils se distinguent des législateurs suisses, qui, dans le Message $d u$ Conseil fédéral du 2 février 1934 concernant le projet de loi fédérale sur les banques, avaient consacré une section entière à l'étude des expériences étrangères (États-Unis, Allemagne, Autriche, Italie, Tchécoslovaquie, Suède, Danemark, Norvège, Angleterre, France, Belgique, Pays-Bas). Cela révèle également que la loi bancaire suisse n'est ellemême pas complètement originale mais emprunte certaines dispositions à d'autres lois étrangères antérieures.

(135) Alors que Dolowitz et Marsh distinguent six types d'acteur dans le processus de policy transfer, dont les groupes de pression, Jacoby estime que le rôle des fonctionnaires étatiques reste prépondérant. Cf. D. DOLOWITZ \& D. MARSH, "Who Learns», op. cit., p. 345; Wade JACOBY, Imitation and Politics. Redesigning Modern Germany, Ithaca, Cornell University Press, 2000, p. 32. 
en ce sens que les plus fervents promoteurs du modèle suisse ne se situent pas parmi les représentants de l'État, mais bien parmi les élites dirigeantes des grandes banques belges.

Du point de vue de l'histoire belge, le processus d'élaboration de l'arrêté royal sur le contrôle bancaire permet d'interroger le caractère progressiste souvent vanté du gouvernement Van Zeeland. Dans le cas du contrôle bancaire, une conception libérale et conservatrice a prévalu, alors même que la configuration politique et le contexte de la crise financière avaient ouvert une fenêtre d'opportunité pour une réforme plus conséquente des activités bancaires. L'influence décisive des cercles bancaires belges a permis de mettre en place un régime de régulation peu contraignant. Dans un processus législatif qui court-circuitait les compétences du Parlement, la surveillance bancaire helvétique, à forte coloration privée et avec une immixtion moindre des instances étatiques, a joué auprès des décideurs belges le rôle d'un modèle à suivre. Cette influence marque les relations entre les banques et l'Etat belges sur le long terme, puisque le cadre de régulation instauré en 1935 sera maintenu pendant près d'un demi-siècle. Disposant des pouvoirs d'intervention très limités, les deux autorités de surveillance nées dans ces conditions ont d'ailleurs connu des difficultés analogues lors de leur mise en place. Dans la perspective de l'historiographie suisse, cette discrète influence helvétique confirme qu'à l'échelle internationale également, la régulation bancaire helvétique de 1934 se distingue par son caractère peu restrictif, à tel point qu'elle est érigée en exemple par un comité de banquiers belges cherchant à limiter l'impact de la puissante vague de réglementation bancaire des années 1930.

\section{RÉSUMÉ}

\section{Thibaud GIDDEY, La surveillance bancaire belge de 1935 façonnée sur le modèle suisse: un discret transfert de politique publique?}

Suite aux graves crises bancaires et financières qui ont secoué la plupart de sociétés occidentales au début des années 1930, on assiste à un foisonnement de réglementations publiques sur les activités bancaires. Parmi les très nombreux pays qui mettent en place des législations bancaires, l'on compte notamment la Suisse et la Belgique. Plus précisément, la loi fédérale suisse sur les banques de novembre 1934 précède de quelques mois l'arrêté royal belge $\mathrm{n}^{\circ} 185$ de juillet 1935 . Alors que le contexte historique et l'élaboration respective de chacune de ces réglementations ont déjà fait l'objet de plusieurs analyses, la comparaison et la question des éventuels liens entre ces deux régimes de surveillance bancaire très proches sont encore largement inexplorées. Une analyse détaillée du processus d'élaboration de la réglementation bancaire belge révèle que la loi suisse a joué un rôle de modèle mis en avant en premier chef par les représentants du monde bancaire. Cette influence helvétique a contribué à façonner la régulation bancaire dans une perspective libérale ; elle répondait ainsi à deux besoins essentiels: limiter l'interventionnisme étatique au minimum, et court-circuiter le pouvoir de la banque centrale. Cette parenté de naissance entre les deux lois bancaires trouve son pendant dans les activités des deux organismes de surveillance créés pour l'occasion, la Commission fédérale des banques à Berne et la Commission bancaire à Bruxelles. Disposant toutes deux de 
moyens d'actions limités, ces deux instances ont connu des parcours analogues au cours de leurs premières années d'activité.

Surveillance bancaire - régulation - banques - État - crise économique - Belgique - Suisse.

\section{SUMMARY}

Thibaud GidDEy, The 1935 Belgian Banking Control System Inspired by the Swiss Model : a Discrete Policy Transfer?

Following the severe financial crisis that shook most of Western societies in the early 1930s, a strong shift towards state regulation on banking activities took place in numerous countries. Among them, both Belgium and Switzerland undertook the implementation of banking laws. More precisely, the Swiss Federal Banking Law of November 1934 was enacted a few months before the Belgian Royal Decree Nr. 185 of July 1935. While the historical context and the development of each of these regulations have been studied individually, a comparative perspective is still lacking. Moreover, the question of the possible links between these two regulatory regimes remains largely unexplored.

A detailed analysis of the Belgian banking regulation process shows that the Swiss law acted as a role model, put forward by the representatives of the banking circles. The Swiss influence played a significant part in shaping the banking regulation in a liberal perspective; thus meeting two essential needs for the Belgian bankers: to limit state intervention to a minimum, and to bypass the authority of the central bank. This relationship between banking laws finds its counterpart in the activities of the two supervision agencies set up in the 1930s, the 'Commission fédérale des banques' in Berne and the 'Commission bancaire' in Brussels. Both bodies featured limited means of actions and experienced similar paths during their first years of operation.

Banking supervision - regulation - state - financial crisis of the 1930s - Belgium - Switzerland.

\section{SAMENVATTING}

Thibaud GIDDEY, Het Belgische bankentoezicht van 1935 gebaseerd op het Zwitserse model: een discrete beleidstransfer?

$\mathrm{Na}$ de ernstige financiële crisis die de westerse samenlevingen teisterde in de vroege jaren 1930, voerden vele landen een overheidsregulering van de bankactiviteiten in. Dat was ook het geval in België en in Zwitserland. De Zwitserse federale bankwet kwam tot stand in november 1934, enkele maanden vóór het Belgische Koninklijk Besluit nr. 185 van juli 1935. De historische context en de totstandkoming van beide wetgevingen werden, elk afzonderlijk, reeds diepgaand onderzocht, maar helaas bleef een comparatieve analyse uit. Bovendien werden de mogelijke banden tussen de beide reguleringsregimes amper onderzocht.

Een gedetailleerde analyse van het Belgische reguleringsproces toont aan dat de Zwitserse wet model stond voor de Belgische ingreep - een model dat naar voren was geschoven door de vertegenwoordigers van de bancaire milieus. De Zwitserse invloed 
speelde immers een grote rol in de vormgeving van de Belgische bankregulering vanuit een liberaal perspectief. Zo werd ingegaan op twee essentiële wensen van de Belgische bankiers: enerzijds de maximale beperking van de overheidstussenkomst en anderzijds het omzeilen van de autoriteit van de centrale bank. Die bijzondere verhouding tussen de twee bankwetten had ook een weerslag op de activiteiten van de twee controle-instanties die in de jaren 1930 werden ingevoerd, namelijk de Commission fédérale des banques in Bern en de Bankcommissie in Brussel. Beide instellingen beschikten over beperkte actiemogelijkheden en functioneerden in de beginjaren ook op een gelijkaardige manier.

Bankcontrole - regulering - Staat - financiële crisis van de jaren 1930 - België - Zwitserland 